\title{
Extension of a coarse grained particle method to simulate heat transfer in fluidized beds
}

\author{
Liqiang Lu ${ }^{1}$, Aaron Morris ${ }^{1}$, Tingwen $\mathrm{Li}^{1,2}$, Sofiane Benyahia ${ }^{1, *}$ \\ 1. National Energy Technology Laboratory, Morgantown, West Virginia 26507, United \\ States
}

2. AECOM, Morgantown, West Virginia, 26505, United States

*Corresponding author: sofiane.benyahia@netl.doe.gov

\begin{abstract}
The heat transfer in a gas-solids fluidized bed is simulated with computational fluid dynamic-discrete element method (CFD-DEM) and coarse grained particle method (CGPM). In CGPM fewer numerical particles and their collisions are tracked by lumping several real particles into a computational parcel. The assumption is that the real particles inside a coarse grained particle (CGP) are made from same species and share identical physical properties including density, diameter and temperature. The parcel-fluid convection term in CGPM is calculated using the same method as in DEM. For all other heat transfer mechanisms, we derive in this study mathematical expressions that relate the new heat transfer terms for CGPM to those traditionally derived in DEM. This newly derived CGPM model is verified and validated by comparing the results with CFD-DEM simulation results and experiment data. The numerical results compare well with experimental data for both hydrodynamics and temperature profiles. The proposed CGPM model can be used for fast and accurate simulations of heat transfer in large scale gas-solids fluidized beds.
\end{abstract}

Key words: Computational fluid dynamics; discrete element method; coarse grained particle method; CFD-DEM; heat transfer

\section{Introduction}

Gas-solids fluidized beds are widely used in industries such as coal gasification and fluidized catalytic cracking. In these applications, heat transfer is of crucial importance for the occurring chemical reactions. It directly influences the reaction kinetics, quality of the final products, and equipment safety. Thus, it is important for chemical engineers to understand the heat transfer mechanisms in fluidized beds. Experimentally, thermocouples are used to detect the temperature distribution in industrial fluidized bed such as circulating fluidized combustion boiler. These are capable of measuring temperatures up to 2600 degrees with a high accuracy. However, these only measure the temperature at a point and need to be in direct contact with the fluids inside the reactor. Another technology is infrared thermography, which can measure the temperature distribution without affecting the flow field. However, this method can only measure the surface temperature and is mostly used in laboratory-scale fluidized beds.

Computer simulation is another powerful tool to study heat transfer, such as the computational fluid dynamic coupled with discrete element method (CFD-DEM) [1-4], which resolves the fluid phase at grid scale and solid phase at particle scale. However, the computation cost of this method is significant due to the large number of particles and small DEM time steps. The coarse grained particle method (CGPM) reduces the computation cost by lumping several real particles into a computation parcel. This method has been used to 
investigate granular flow $[5,6]$ and hydrodynamics in gas-solids flows such as pneumatic conveying[7], bubbling fluidized bed [8,9], cyclone separator [10], circulating fluidized bed (CFB) riser [11], full loop of CFB [12] and chemical leaching process of rare earth elements [13]. To our knowledge, heat transfer models are not included in any of the published CGPM. Due to the importance of heat transfer in many industrial applications, this research proposes a heat transfer model for CGPM. The proposed heat transfer model is an extension of models designed for real particles in order to accommodate the concept of computational parcels. To achieve this goal, we first describe the details of heat transfer models for DEM and their extension to CGPM. Then we compare the results obtained for CGPM and those obtained by using DEM as well as experimental data. Conclusions are then drawn based on this comparison.

\section{Method}

In CGPM, several real particles are lumped into a coarse grained or computational parcel (CGP). The drag, gravity, and pressure forces are calculated at real particle scale following the same process in traditional CFD-DEM while the collision forces are calculated using the diameter of CGPs, $d_{\mathrm{CGP}}$. This new diameter is calculated as $d_{\mathrm{CGP}}=d_{p} W^{1 / 3}=k d_{p}$, where $W$ is the statistical weight representing the number of real particles in a CGP and $k$ is length scale ratio. In this section, the hydrodynamic model of CGPM is first briefly reviewed, and then we focus on the newly developed heat transfer models for CGPM.

\subsection{Fluid governing equations}

The gas phase is described by the volume-averaged Navier-Stokes equations [14],

$$
\begin{gathered}
\frac{\partial\left(\varepsilon_{\mathrm{f}} \rho_{\mathrm{f}}\right)}{\partial t}+\left(\nabla \cdot \varepsilon_{\mathrm{f}} \rho_{\mathrm{f}} \mathbf{u}_{\mathrm{f}}\right)=0 \\
\frac{D\left(\varepsilon_{\mathrm{f}} \rho_{\mathrm{f}} \mathbf{u}_{\mathrm{f}}\right)}{D t}=\nabla \cdot \overline{\bar{S}}_{\mathrm{f}}+\varepsilon_{\mathrm{f}} \rho_{\mathrm{f}} \mathbf{g}-\mathbf{I}
\end{gathered}
$$

Where $\varepsilon_{f}$ is the volume fraction of fluid, $\rho_{f}$ is the density of fluid, $\mathbf{u}_{f}$ is the velocity of fluid, and

I is the drag source term. $\overline{\bar{S}}_{\mathrm{f}}$ is the fluid phase stress tensor given by

$$
\overline{\bar{S}}_{\mathrm{f}}=-P_{\mathrm{f}} \overline{\bar{I}}+\bar{\tau}_{\mathrm{f}}
$$

Where $P_{\mathrm{f}}$ is the fluid phase pressure. Also, $\overline{\boldsymbol{\tau}}_{\mathrm{f}}$ is the fluid phase shear stress tensor

$$
\begin{gathered}
\bar{\tau}_{\mathrm{f}}=2 \mu_{\mathrm{f}} \overline{\bar{D}}_{\mathrm{f}}+\lambda_{\mathrm{f}} \nabla \cdot \operatorname{tr}\left(\overline{\bar{D}}_{\mathrm{f}}\right) \overline{\bar{I}} \\
\overline{\bar{D}}_{\mathrm{f}}=\frac{1}{2}\left[\nabla \mathbf{u}_{\mathrm{f}}+\left(\nabla \mathbf{u}_{\mathrm{f}}\right)^{T}\right]
\end{gathered}
$$

Where $\overline{\bar{D}}_{\mathrm{f}}$ is the strain rate tensor, and $\mu_{\mathrm{f}}$ and $\lambda_{\mathrm{f}}$ are the dynamic and second coefficient of viscosity of the fluid phase.

The inter-phase momentum transfer term on fluid cell $c$ can be calculated as

$$
\mathbf{I}^{c}=\frac{1}{v_{c}} \sum_{i=1}^{N_{p}} \frac{1}{6} \pi d_{\mathrm{p}}^{3} W_{p}\left(\nabla P_{\mathrm{f}}\left(\mathbf{x}^{i}\right)+\frac{\beta^{i}}{1-\varepsilon_{\mathrm{f}}}\left(\mathbf{v}_{\mathrm{f}}\left(\mathbf{x}^{i}\right)-\mathbf{v}_{\mathrm{p}}^{i}\right)\right) K\left(\mathbf{x}^{i}, \mathbf{x}_{c}\right)
$$


Where $v_{c}$ is the volume of cell $c, N_{p}$ is number of particles influence cell $c, W_{p}$ is statistic weight of particle $p, \beta$ is drag coefficient of particle $i$ in cell $c, \mathbf{v}_{\mathbf{f}}\left(\mathbf{x}^{i}\right)$ is the fluid velocity interpolated at particle $i$ and $K$ is the interpolation weight of particle $i$ to cell $c$. In gas-solids flows, the drag coefficient should be corrected by considering the heterogeneous structures [15-18]. In this study we use the drag correlation proposed by Beetstra et al. [19] expressed as

$$
\begin{gathered}
F=\frac{10\left(1-\varepsilon_{f}\right)}{\varepsilon_{f}^{2}}+\varepsilon_{f}^{2}\left(1+1.5\left(1-\varepsilon_{f}\right)^{0.5}\right) \\
+\frac{0.413 \operatorname{Re}_{p}}{24 \varepsilon_{f}^{2}}\left[\frac{\varepsilon_{f}^{-1}+3 \varepsilon_{f}\left(1-\varepsilon_{f}\right)+8.4 \operatorname{Re}_{p}^{-0.343}}{1+10^{3\left(1-\varepsilon_{f}\right)} \operatorname{Re}_{p}^{-0.5-2\left(1-\varepsilon_{f}\right)}}\right] \\
\beta=\frac{18 \mu \varepsilon_{f}\left(1-\varepsilon_{f}\right) F}{d_{p}^{2}}
\end{gathered}
$$

Where

$$
R e_{\mathrm{p}}=\frac{\varepsilon_{\mathrm{f}} \rho_{\mathrm{f}}\left|\mathbf{v}_{\mathrm{f}}-\mathbf{v}_{\mathrm{p}}\right| d_{\mathrm{p}}}{\mu_{\mathrm{f}}}
$$

\subsection{Equations of motion for particles}

The Lagrangian method is used to track the motion of fewer computational parcels. For each parcel, the momentum equation takes the form:

$$
m_{\mathrm{p}} \frac{d \mathbf{v}_{\mathrm{p}}}{d t}=m_{\mathrm{p}} \mathbf{g}-\frac{\pi}{6} d_{p}^{3} \nabla P_{f}+\frac{\beta\left(\mathbf{v}_{\mathrm{f}}(\mathbf{x})-\mathbf{v}_{\mathrm{p}}\right)}{1-\varepsilon_{f}} \frac{\pi}{6} d_{p}^{3}+\mathbf{F}_{\mathrm{c}}
$$

Where $m_{\mathrm{p}}$ is the mass of the real particle and $d_{\mathrm{p}}$ is its diameter. On the right-hand side, the forces considered include the gravity force, pressure gradient force, drag force and the contact force $\left(\mathbf{F}_{\mathrm{c}}\right)$. For the first three terms, they can be calculated following the same process as in traditional CFD-DEM. The contact force is calculated by the discrete element method (DEM) using $d_{\mathrm{CGP}}$ as collision diameter, the force is then divided by statistic weight to represent the force on a real particle:

$$
\mathbf{F}_{\mathrm{c}}=\sum_{j=1, j \neq i}^{N}\left(\mathbf{F}_{i j}^{\mathrm{n}}+\mathbf{F}_{i j}^{\mathrm{t}}\right) / W
$$

As for particle rotation, the moment of inertia $I$ is calculated by $m_{p} d_{p}{ }^{2} / 10$, and is $W^{5 / 3}$ times smaller than the inertia based on coarse grained particle. Thus, the torque on each tracked particle should be calculated as:

$$
\mathbf{T}=\sum_{j=1, j \neq i}^{N}\left(L \mathbf{n} \times \mathbf{F}_{i j}^{t}\right) / W^{5 / 3}
$$

Where $L$ is the distance from particle center to contact point. The details of the DEM model can be found in the MFIX-DEM documentation [20] and validation studies in [21, 22]. All the 
details of the CGPM hydrodynamic model and its validation can be found in a recent publication [13].

Upon collisions, the energy dissipation in the coarse-graining system must de same as the original system with real particles $[7,23]$, and so a new coefficient of restitution is derived from kinetic theory of granular flow [24, 25] as shown below [11]:

$$
e_{\mathrm{CGP}}=\sqrt{1+\left(e_{\mathrm{p}}^{2}-1\right) W^{1 / 3}}
$$

\subsection{Heat transfer equations for particles}

In fluidized beds, the heat transfer mechanisms for particles include particle-fluid convection, particle-fluid-particle conduction, particle-particle conduction, particle-fluid-wall conduction, particle-wall conduction. The radiative heat transfer is not treated here because it is not of interest to this particular research and is, thus, left for future development. Sun and Chen [26] proposed a model for particle-particle and particle-wall conduction heat transfer. The maximum contact area is calculated based on Hertzian theory with heat transfer occurring only along one-dimensional line perpendicular to the contact area between two particles. The heat transfer during each contact is then calculated using an analytical solution which can be easily implemented in a numerical code. Instead of using the maximum contact area, Batchelor and O'Brien [27] use the instantaneous normal contact force to calculate the contact radius and the heat transfer is integrated during each time-step. This model is generalized further by using the geometric configuration of the two particles to obtain the contact area and has been implemented in MFIX-DEM [28]. For particle-fluidparticle and particle-fluid-wall conduction, Cheng, Yu and Zulli [29] developed two structurebased analytic models by assuming heat transfer is restricted to the region of a double tapered cone. This model has been used by Zhou, Yu and Zulli [30] to study the heat transfer in gas-solids fluidized bed. Rong and Horio [31] proposed another model by assuming each particle is surrounded by a gas layer and the heat transfer initiates when the gas layer overlaps with wall boundaries or other particles. This model is also available in our software MFIX-DEM. The particle-fluid convection is proportional to temperature difference, surface area, and convective heat transfer coefficient. The convective coefficient is related to Nusslet number which expresses the ratio of convective to conductive heat transfers. These terms are described and developed further in this section.

The conservation equation for heat transfer of particle $i$ surrounded by fluid and particles includes: particle-fluid convection $\left(Q_{i, \text { conv }}\right)$, particle-particle and particle-wall conductions $\left(Q_{i, j}\right.$, $\left.Q_{i, \text { wall }}\right)$ and is expressed by the following equation:

$$
m_{i} c_{p, i} \frac{d T_{i}}{d t}=Q_{i, c o n v}+Q_{i, w}+\sum_{j=1}^{K_{i}} Q_{i, j}
$$

The convection term is calculated by:

$$
Q_{i, \text { conv }}=h_{i, \text { conv }} \pi d_{p}^{2}\left(T_{f}-T_{i}\right)
$$

Where the heat transfer coefficient $h_{i, c o n v}$ is a function of particle Nusselt number, and the correlation given by Gunn [32] is used: 


$$
\begin{gathered}
N u_{i}=\left(7-10 \varepsilon_{f}+5 \varepsilon_{f}^{2}\right)\left(1.0+0.7 \operatorname{Re}_{i}^{0.2} \operatorname{Pr}^{0.33}\right) \\
+\left(1.33-2.4 \varepsilon_{f}+1.2 \varepsilon_{f}^{2}\right) \operatorname{Re}_{i}^{0.7} \operatorname{Pr}^{0.33} \\
N u_{i}=\frac{h_{i, \text { conv }} d_{p}}{k_{f}}, \quad \operatorname{Pr}_{i}=\frac{\mu_{f} C_{f}}{k_{f}}
\end{gathered}
$$

Where $d_{p}$ and $k_{f}$ are the particle diameter and fluid thermal conductivity. $\operatorname{Re}_{i}$ is the local Reynolds number of particle $i . C_{f}$ is the specific heat of fluid.

As shown in Fig. 1, the model introduced by Rong and Horio [31] is used in this research. They hypothesize that each particle is surrounded by a gas layer with a constant thickness, $r_{\text {len; }}$; particle-fluid-wall heat transfer initiates when the gas layer intersects with the wall; heat transfer occurs across paths parallel to the axis joining the particle's center and the wall; a uniform gas layer, with a constant thickness $s$, separates the particle and the wall; the temperature is assumed uniform inside a particle. Thus, this term can be calculated as

$$
Q_{i, w}^{p f w}=2 \pi k_{g}\left(T_{w}-T_{i}\right) \int_{r_{\text {in }}}^{r_{\text {out }}} \frac{r}{l} d r
$$

Where $k_{\mathrm{g}}$ is the gas thermal conductive coefficient, $T_{w}$ and $T_{i}$ are the temperatures of wall and particle, $I$ is the distance between particle and wall, the integration from $r_{\text {in }}$ to $r_{\text {out }}$ delineating the region of particle-fluid-wall conduction.

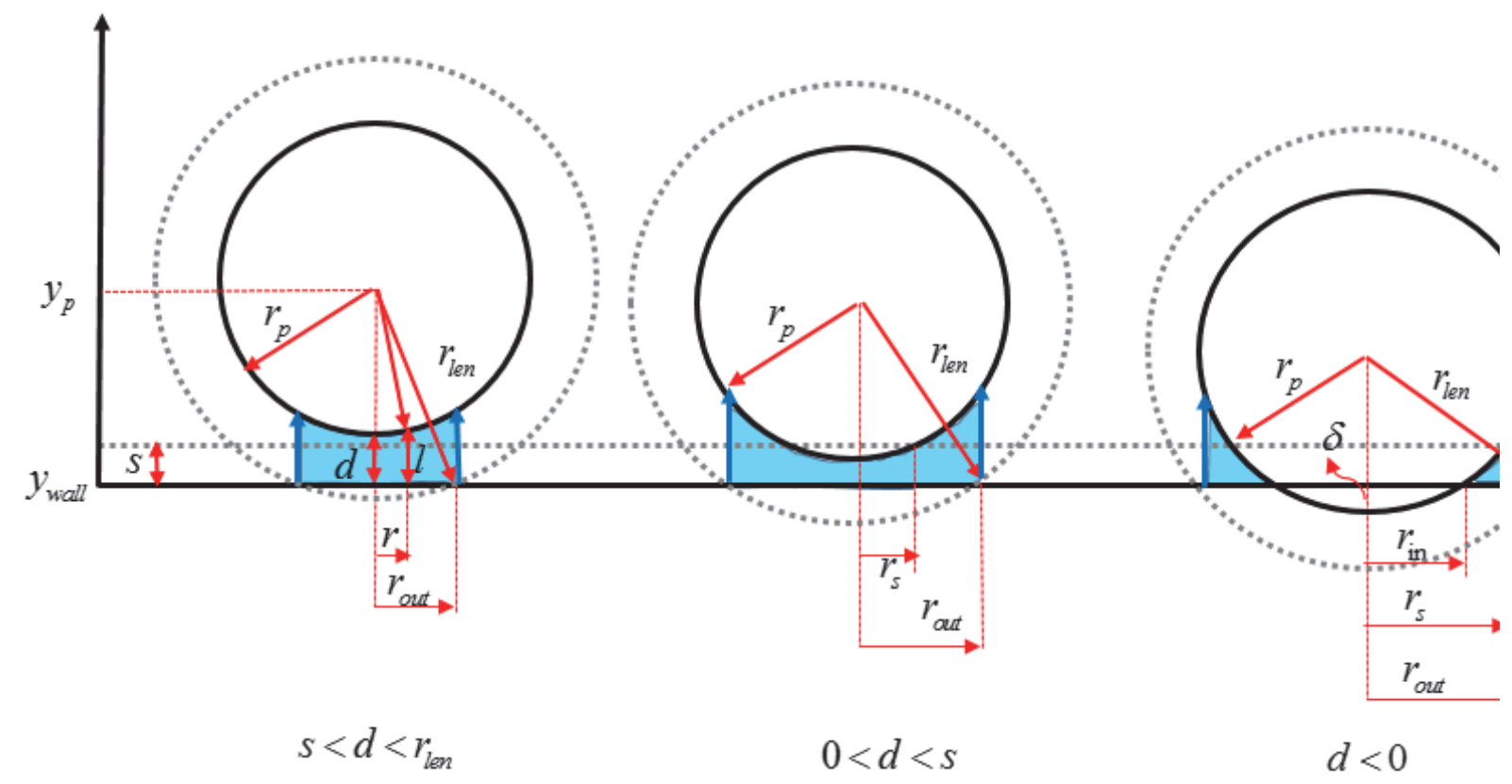

Fig. 1 Particle-fluid-wall heat transfer model. From (a) to (c), the particle is getting closer to the wall. In case (a) only the gas layer is in contact with the wall and the distance is larger than $s$; in case (b) the distance is smaller than $s$; in case (c) the particle is in contact with wall

In CGPM, the collision between particle and wall is calculated using $d_{\text {CGP }}$ as collision diameter, and the distance / will be different from the original system when a particle collides with the wall. Eq.18 cannot be used directly in the simulation. To make sure the particle-fluidwall transferred thermal energy is same between CGP system and original system, we use the following non-dimensional form of Eq. 18 to derive the particle-fluid-wall heat transfer for 
CGP.

$$
\bar{Q}_{i, w}^{p f w}=\frac{Q_{i, w}^{p f w}}{2 \pi k_{g}\left(T_{w}-T_{i}\right) r_{p}}=\int_{\bar{r}_{\text {in }}}^{\bar{r}_{\text {out }}} \frac{\bar{r}}{\bar{l}} d \bar{r}
$$

The distance / will decrease as the particle get closer to the wall, and when the particle is in contact with the wall, or $I=0$, a singularity will appear in this equation. This singularity is solved by assuming a minimum distance $s$ between particle and wall. Thus, the integral can be converted to three non-singular functions according to particle-wall distance.

$$
\bar{Q}_{i, w}^{p f w}=\int_{\bar{r}_{\text {in }}}^{\bar{r}_{\text {out }}} \frac{\bar{r}}{\bar{l}} d \bar{r}= \begin{cases}\int_{0}^{\bar{r}_{\text {out }}} \frac{\bar{r}}{\bar{l}} d \bar{r} & \bar{s}<\bar{d}<\bar{r}_{\text {len }} \\ \int_{0}^{\bar{r}_{s}} \overline{\bar{r}} d \bar{r}+\int_{\bar{r}_{\text {in }}}^{\bar{r}_{\text {out }}} \overline{\bar{r}} \frac{\overline{\bar{l}}}{\bar{s}} & 0<\bar{d}<\bar{s} \\ \int_{\bar{r}_{\text {in }}} \frac{\bar{r}}{\bar{s}} d \bar{r}+\int_{\bar{r}_{s}}^{\bar{r}_{\text {out }}} \frac{\bar{r}}{\bar{l}} d \bar{r} & \bar{d}<0\end{cases}
$$

For reader's convenience, the integration results and the geometrical relationships among $r_{s}, r_{\text {in }}, r_{\text {out }}, l$ and $r$ are attached in the appendix. The non-dimensional form particlefluid-wall heat transfer for the original system and coarse grained system can be written as

$$
\begin{gathered}
\bar{Q}_{i, w}^{p f w r p}=f\left(\bar{\delta}_{r p}, \bar{r}_{l e n, r p}, \bar{s}_{r p}\right) \\
\bar{Q}_{i, w}^{p f w C G P}=f\left(\bar{\delta}_{C G P}, \bar{r}_{l e n, C G P}, \bar{s}_{C G P}\right)
\end{gathered}
$$

Therefore, the non-dimensional particle-fluid-wall heat transfer is only related to nondimensional particle wall distance, gas layer, and particle wall minimum distance. To ensure the heat conduction is same for both systems, we must assume the non-dimensional minimum distance and gas layer length are same for the original system and coarse grained system.

$$
\bar{r}_{l e n, C G P}=\bar{r}_{l e n, r p}, \quad \bar{s}_{C G P}=\bar{s}_{r p}
$$

This relationship is also expressed graphically in Fig. 2. It can be seen that when the particle is not in contact with wall $\left(d_{n}<0\right)$, the heat transfer increases with the decrease of particle-wall distance. However, when the particle contacts the wall, the heat transfer keeps almost constant. Thus, we only need to ensure the same non-dimensional particle-wall distance before a particle contacts the wall. 


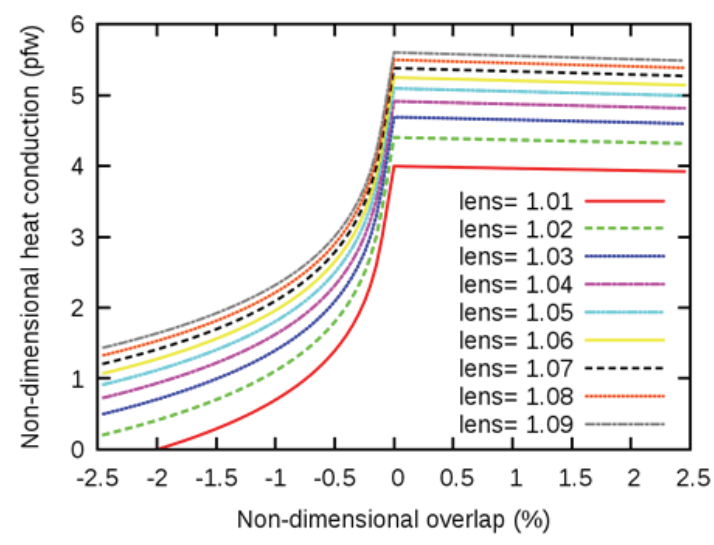

(a) $\bar{s}=0.001$

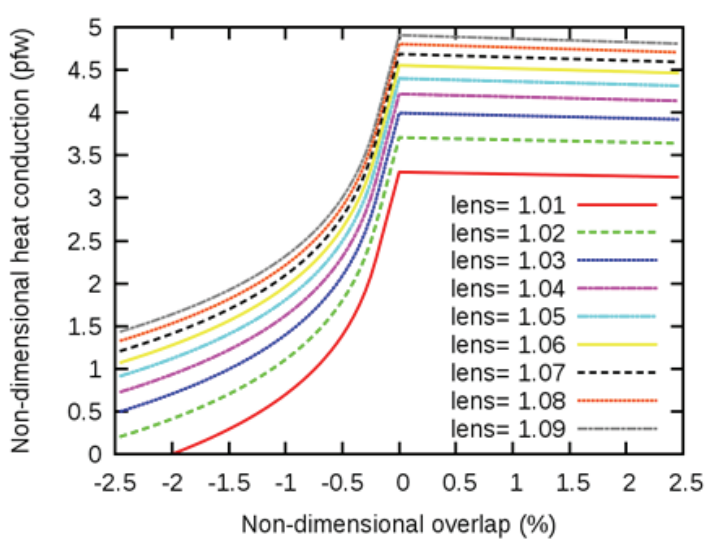

(b) $\bar{s}=0.002$

Fig. 3 Non-dimensional particle-fluid-wall heat transfer dependence on non-dimensional overlap, gas layer and minimum distance

For non-dimensional particle wall distance, as shown in Fig. 4, we assume the particle center of the coarse grained system is same to the averaged center of the original system. Thus, the distance to the wall is same for both original system and coarse grained system.

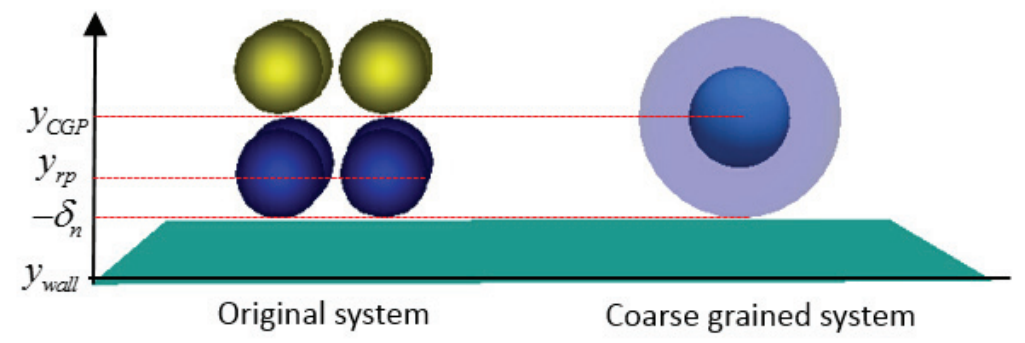

Fig. 4 Relationship of wall distance for original system and coarse grained system. For original system only $k^{2}$ particles (blue colored particles) are performing particle-fluid-wall heat transfer.

The CGP diameter $d_{\mathrm{CGP}}$ is used for both collision dynamics and particle-fluid-wall heat transfer. Thus the relationship of non-dimensional particle wall distance in original system and coarse grained system is:

$$
\bar{\delta}_{r p}=k \bar{\delta}_{C G P}
$$

Then, the non-dimensional form particle-fluid-wall heat transfer for coarse grained system can be written as

$$
\bar{Q}_{i, w}^{p f w C G P}=f\left(k \bar{\delta}_{C G P}, \bar{r}_{l e n, C G P}, \bar{s}_{C G P}\right)
$$

For the original system only $k^{2}$ particles (blue colored particles in Fig. 4) are performing particle-fluid-wall heat transfer. Thus, the average value should be

$$
\bar{Q}_{\text {avg }}^{p f w r p}=\sum_{i=1}^{W} \bar{Q}_{i, w}^{p f, w r p} W=\bar{Q}_{i, w}^{p f w r p} k
$$

Considering $\bar{Q}_{i, w}^{p f w r p}=\bar{Q}_{i, w}^{p f, w C G .}$ and Eq.19, we can get: 


$$
Q_{\text {avg }}^{p f w r p}=Q_{a v g}^{p f, w C G P} / k^{2}
$$

This equation means that the particle-fluid-wall heat conduction in CGPM is $k^{2}$ times larger than in the original system. To make sure that the two systems give the same result, the calculated particle-fluid-wall heat conduction in CGPM must be rescaled by a factor of $1 / k^{2}$.

According to [30], in fluidized beds particle-particle conduction through the contacting area accounts for less than $1 \%$ of the total conduction term for particles with a thermal conductivity of $0.08 \mathrm{~W} /(\mathrm{m} \mathrm{K})$ and about $15 \%$ for particles with a thermal conductivity of 30 $\mathrm{W} /(\mathrm{m} \mathrm{K})$. Considering the total conduction term is about $1 / 4$ times of the convection term, the particle-particle conduction through the contacting area accounts for less than $3 \%$ of the total particle heat transfer even for particles with a high thermal conductivity. From an engineering point of view, this term may be neglected. However and for completeness, we derive the conductive heat transfer model in the section below.

For particle-wall contact conduction term, the model proposed by Batchelor and O'Brien [27] is adopted here. When a particle is in contact with wall, the conduction heat transfer can be calculated as

$$
Q_{i}^{p w}=\frac{4 r_{c}\left(T_{w}-T_{i}\right)}{\left(1 / k_{p i}+1 / k_{w}\right)}
$$

Where $r_{\mathrm{c}}$ is the contact radius, $k_{\mathrm{pi}}$ and $k_{\mathrm{w}}$ are thermal conductive coefficients of particle and wall, $T_{w}$ and $T_{i}$ are the temperatures of wall and particle, and $r_{c}$ can be calculated by the following equation

$$
r_{c}=\sqrt{r_{p}^{2}-\left(r_{p}-\delta\right)^{2}}=\sqrt{2 r_{p} \delta-\delta^{2}}
$$

For original system and coarse grained system, $r_{c}$ can be written as:

$$
\begin{gathered}
r_{c}^{r p}=\sqrt{2 r_{r p} \delta_{r p}-\delta_{r p}^{2}} \\
r_{c}^{C G P}=\sqrt{2 r_{C G P} \delta_{C G P}-\delta_{C G P}^{2}}
\end{gathered}
$$

It is difficult to derive a precise relationship between $\delta_{r p}$ and $\delta_{C G P}$. However, since the particlewall contact conduction is small compared with particle-fluid-wall, a very simple assumption is used here. Therefore, we assume that the maximum overlap is representative of the otherwise variable overlap during the particle-wall collision. Since this collision is modeled here by a massspring-dashpot system, as derived in [33], the maximum overlap can be expressed as:

$$
\delta_{\max }=\sqrt{\frac{v_{0}^{2} m_{\text {eff }}}{k_{n}}} f(e)
$$

Where $v_{0}$ is the initial collision velocity, $k_{n}$ is spring constant and $m_{\text {eff }}$ is effective mass. The expression of $f(e)$ can be evaluated by 


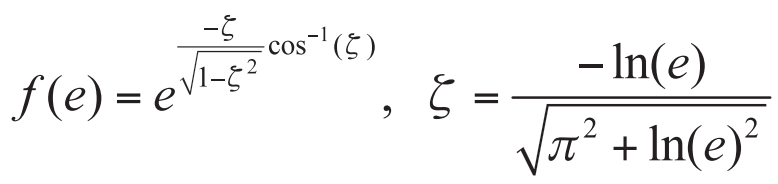

Since the same spring constant $k_{n}$ used in DEM is also used in our CGPM simulation, thus the overlap is calculated as:

$$
\delta_{C G P}=\delta_{r p} \sqrt{k^{3}} \frac{f\left(e_{C G P}\right)}{f\left(e_{r p}\right)}
$$

So, the contact radius of the CGP is:

$$
r_{c}^{C G P}=k^{5 / 4}\left(\frac{f\left(e_{C G P}\right)}{f\left(e_{r p}\right)}\right)^{1 / 2} \sqrt{2 r_{r p} \delta_{r p}-k^{1 / 2} \frac{f\left(e_{C G P}\right)}{f\left(e_{r p}\right)} \delta_{r p}^{2}}
$$

In DEM simulation, the particle-wall overlap is usually much smaller than the collision radius. The contact radius of coarse grained system can, therefore, be approximated as:

$$
r_{c}^{C G P}=k^{5 / 4}\left(\frac{f\left(e_{C G P}\right)}{f\left(e_{r p}\right)}\right)^{1 / 2} r_{c}^{r p}
$$

The error introduced here depends on restitution coefficient of the original system and scale ratio $k$. We also need to consider the particle-wall collision frequency as shown in Fig. 4. Thus, the equation for particle-wall contact conduction is

$$
Q_{r p}^{p w}=\frac{Q_{C G P}^{p w}}{k^{9 / 4}}\left(\frac{f\left(e_{r p}\right)}{f\left(e_{C G P}\right)}\right)^{1 / 2}
$$

Similar to particle-wall heat conduction, the particle-particle conduction involves particlefluid-particle conduction and particle-particle contact conduction. The relationship between the coarse grained system and the original system can be derived by following the same procedure as for particle-wall conduction. Considering a CGP representing $k^{3}$ number of real particles, only $k^{2}$ real particles on the surface can transfer heat to other CGP either through particle-fluid-particle conduction or contact conduction. Thus, the relationship derived for particle-wall conduction is still applicable to particle-particle conduction. That is

$$
\begin{gathered}
Q_{r p}^{p f p}=Q_{r p}^{p f p} k^{2} \\
Q_{r p}^{p p}=\frac{Q_{C G P}^{p p}}{k^{9 / 4}}\left(\frac{f\left(e_{r p}\right)}{f\left(e_{C G P}\right)}\right)^{1 / 2}
\end{gathered}
$$

In DEM simulation, $r_{\mathrm{c}}$ is usually overestimated due to the use of a much smaller Yong's modules. Thus, it is difficult to calculate this term precisely even in DEM. Zhou, Yu and Zulli [34] proposed a correction coefficient $c$ to reduce this overestimation for DEM simulation with Hertzian model. This correction has been extended to linear spring-dashpot model in MFIXDEM as 


$$
r_{c, 0}=\left(\frac{k_{n} r_{c}}{k_{n, 0}}\right)^{2 / 3}
$$

Where $r_{\mathrm{c}, 0}$ is the reduced contact radius. $k_{n}$ is the spring constant used in the simulation, $k_{n, 0}$ is spring constant derived from Yong's modules of particles through:

$$
\begin{aligned}
& k_{n, 0}=\frac{4}{3} \sqrt{r_{e f f}} E_{e f f} \\
& \frac{1}{r_{e f f}}=\frac{1}{r_{i}}+\frac{1}{r_{j}} \\
& \frac{1}{E_{e f f}}=\frac{1-v_{i}^{2}}{E_{i}}+\frac{1-v_{j}^{2}}{E_{j}}
\end{aligned}
$$

Where $r_{\mathrm{i}}$ and $r_{j}$ are particle radius of particles $i$ and $j . E_{\mathrm{i}}$ and $E_{j}$ are Yong's modules of particles $i$ and $j . v_{i}$ and $v_{j}$ are poisson ratios of particles $i$ and $j$.

Finally for the fluid phase, the conservation equation of thermal energy is expressed as:

$$
\varepsilon_{\mathrm{f}} \rho_{\mathrm{f}} C_{p f}\left(\frac{\partial T_{f}}{\partial t}+\mathbf{u}_{\mathrm{f}} \cdot \nabla T_{f}\right)=-\nabla \cdot \mathbf{q}_{g}+S_{p f}+S
$$

Where $S_{p f}$ is total heat transfer from solid particles and $S$ is any other source terms, such as heat generated or consumed due to chemical reactions. The conductive heat flux is described by Fourier's law:

$$
\dot{q}_{f}=-\varepsilon_{f} k_{f} \nabla T_{f}
$$

\section{Simulation parameters}

To verify the method proposed in Sect. 2, a gas-solids fluidized bed is simulated. The experimental setup by Patil et al. [35] is shown in Fig. 4. In this fluidized bed, glass beads initially at $90{ }^{\circ} \mathrm{C}$ are fluidized by a cooler nitrogen gas at $20{ }^{\circ} \mathrm{C}$. The front plate of the bed is made of sapphire glass so that the fluidized bed is visible for camera recording. The back and side walls are made of aluminum. Experimentally, there is a circular nozzle with a diameter of $1.3 \mathrm{~cm}$ at the bottom of the bed. In the simulation, however, this area is blocked with a rectangular $1.14 \mathrm{~cm} \times 1$ $\mathrm{cm}$ surface. There is an exception for the case CGPM-27, where the CFD grid is too large to resolve this small rectangular patch. Therefore and to ensure the same fluid flow rate through the bottom inlet, the gas velocity for case CGPM-27 is reduced from 1.33 to $1.20 \mathrm{~m} / \mathrm{s}$. Other simulation parameters and physical properties are listed in Table 1-2. 


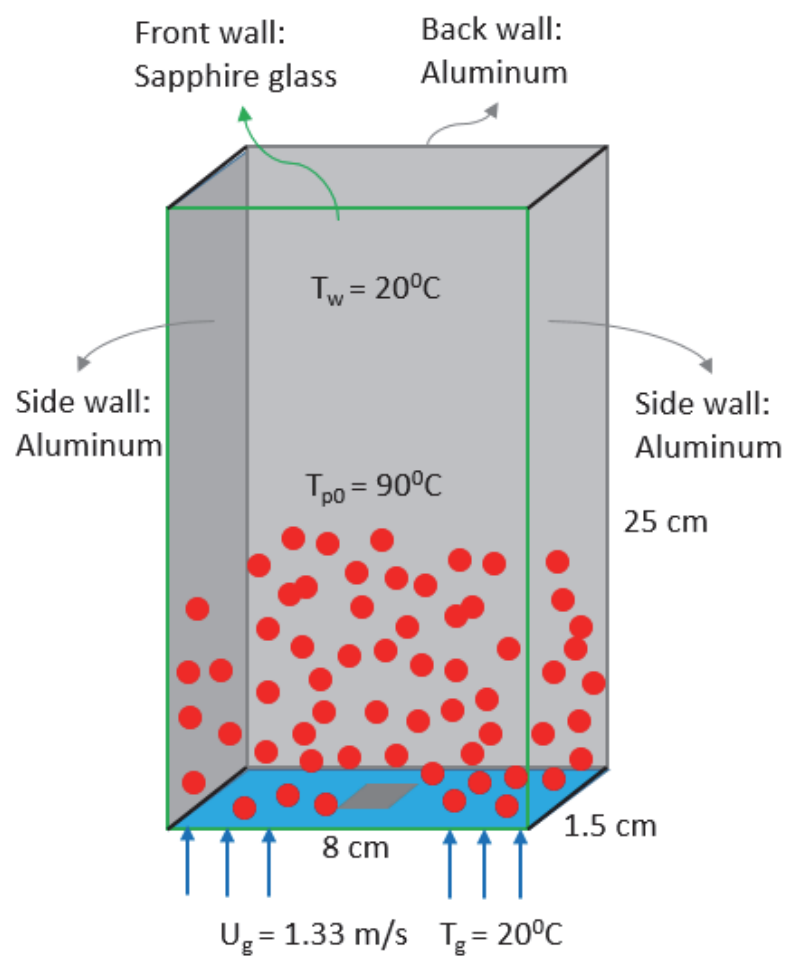

Fig. 6 Details of the experimental setup of Patil et al. [35] as adapted to the CFD simulation.

Table 1 Simulation parameters and physical properties

\begin{tabular}{ll}
\hline Parameter or property & Value \\
\hline Particle diameter, $d_{\mathrm{p}}(\mathrm{m})$ & $1.0 \times 10^{-3}$ \\
Particle density, $\rho_{\mathrm{p}}\left(\mathrm{kg} / \mathrm{m}^{3}\right)$ & 2500 \\
Particle Spring constant, $k_{\mathrm{n}}(\mathrm{N} / \mathrm{m})$ & $1.0 \times 10^{2}$ \\
Particle Restitution coefficient, $e$ & 0.97 \\
Particle friction coefficient, $\mu$ & 0.30 \\
Particle heat capacity, $C_{\mathrm{p}}(\mathrm{J} / \mathrm{kg} \mathrm{K})$ & 840 \\
Particle thermal conductivity, $\mathrm{k}_{\mathrm{p}}(\mathrm{W} / \mathrm{m} \mathrm{K})$ & 1.40 \\
Aluminum thermal conductivity, $\mathrm{k}_{\mathrm{Al}}(\mathrm{W} / \mathrm{m} \mathrm{K})$ & 238 \\
Sapphire thermal conductivity, $\mathrm{k}_{\mathrm{Al}}(\mathrm{W} / \mathrm{m} \mathrm{K})$ & 40 \\
Fluid heat capacity, $C_{\mathrm{f}}(\mathrm{J} / \mathrm{kg} \mathrm{K})$ & 1010 \\
Fluid viscosity, $\mu_{\mathrm{f}}(\mathrm{kg} / \mathrm{m} \mathrm{s})$ & $2.0 \times 10^{-5}$
\end{tabular}


Table 2 Simulation parameters for CFD-DEM and CGPM

\begin{tabular}{|c|c|c|c|}
\hline & CFD-DEM & CGPM-W8 & CGPM-W27 \\
\hline CFD grids, $N_{x} \times N_{y} \times N_{z}$ & $35 \times 110 \times 6$ & $35 \times 110 \times 6$ & $16 \times 50 \times 3$ \\
\hline CFD time step, $\Delta t(\mathrm{~s})$ & $1.0 \times 10^{-4}$ & $5.0 \times 10^{-4}$ & $5.0 \times 10^{-4}$ \\
\hline Number of CGP, $N_{C G P}$ & 57296 & 7162 & 2122 \\
\hline Statistic weight, $W$ & 1 & 8 & 27 \\
\hline Solid time step, $\Delta t$ (s) & $1.27 \times 10^{-5}$ & $1.44 \times 10^{-5}$ & $2.64 \times 10^{-5}$ \\
\hline Gas layer diameter, $r_{l e n}(\mathrm{~m})$ & $1.04 \times 10^{-3}$ & $2.08 \times 10^{-3}$ & $3.12 \times 10^{-3}$ \\
\hline $\begin{array}{l}\text { Minimum conduction distance, } s \\
(\mathrm{~m})\end{array}$ & $1.0 \times 10^{-6}$ & $2.0 \times 10^{-6}$ & $3.0 \times 10^{-6}$ \\
\hline Fluid inlet velocity, $\bigcup_{f}(\mathrm{~m} / \mathrm{s})$ & 1.33 & 1.33 & 1.20 \\
\hline
\end{tabular}

\section{Results and discussion}

\subsection{Predictions of the fluidization behavior}

Since heat transfer is directly coupled with hydrodynamics, the predictions of particle distribution is first investigated. Fig. 5 shows the instantaneous particle distributions predicted for different $W$ as the simulation progresses. Reasonable similarity of flow patterns for CFD-DEM and CGPM-8 were predicted. This figure shows a downward flow of particles near the wall boundaries while a high velocity upward flow of particles is clearly visible at the core of the bed. All the three cases simulated here capture a similar bubbling behavior.

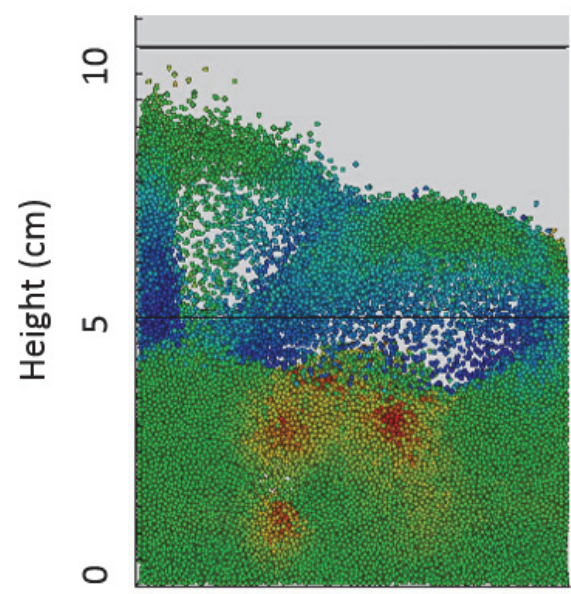

CFD-DEM

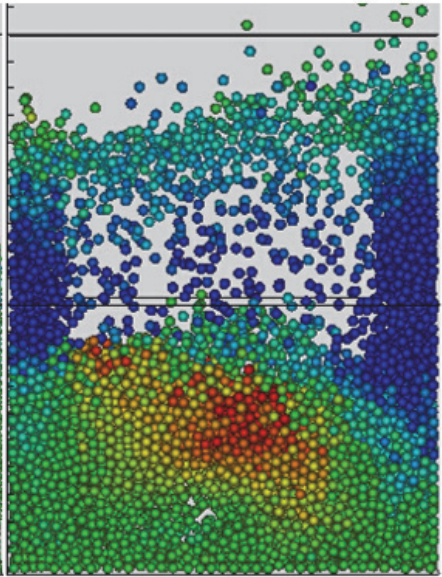

CGPM-8

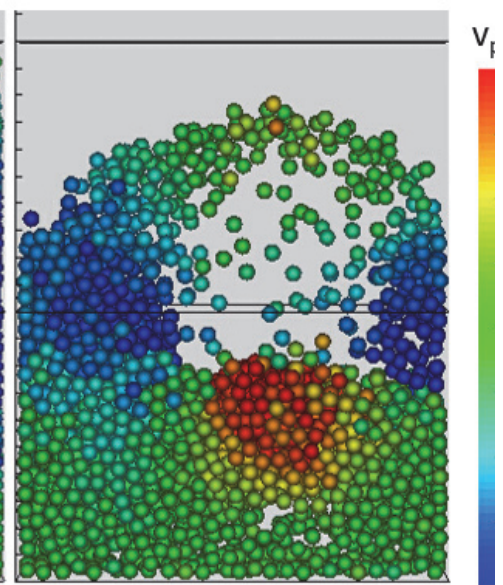

CGPM-27

(a) Time $=3.5 \mathrm{~s}$ 


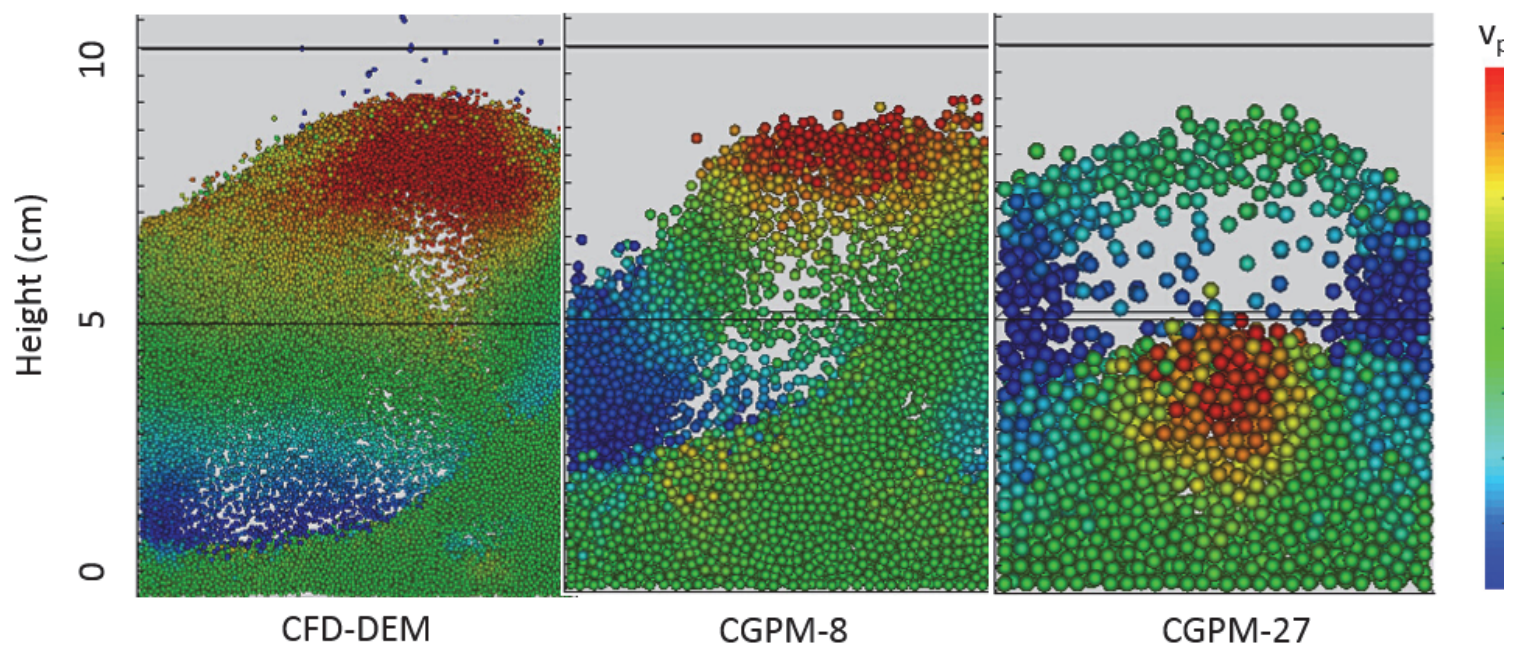

(b) Time $=5.5 \mathrm{~s}$

Fig. 7 Particles distribution at two instants using different statistic weights (colored by vertical velocity of particles)

Fig. 6 (a) shows a comparison of the time-averaged experimental particles volumetric flux in axial direction with simulation results at $2.3 \mathrm{~cm}$ above the gas inlet distributor. This comparison shows that the CFD-DEM radial profile is in excellent agreement with those published previously and using the same computational approach [35]. The numerical results obtained using CGPM-8 approximation also compare well with CFD-DEM and experimental data. However, for CGPM-27, the flux near the center of the fluidized bed is higher than that captured by both CFD-DEM and experimental data. This difference may be due to the coarser computational grid for CFD used in this particular case (CGPM-27). In fact, the collision diameter of these parcels is $3 \mathrm{~mm}$ and, to ensure the CFD grid size is larger than parcel diameter, the grid size in this case is increased to $5 \mathrm{~mm}$. Thus, this grid size is too coarse to resolve the rectangle area shown in Fig. 4.

Fig. 6 (b) and Fig. 6 (c) show the radial profiles of solids volume fraction and axial velocity. Note that all the computational data were averaged from $5.0 \mathrm{~s}$ to $8.0 \mathrm{~s}$ with a frequency of $10 \mathrm{~Hz}$. Also, note that no experimental data were available for comparison. Recall that for the case CGPM-27, the inlet velocity was decreased from 1.33 to $1.20 \mathrm{~m} / \mathrm{s}$ due to an increase in inlet flow area. Thus, some of the disagreement between DEM and the coarsest CGPM may be due to this small difference in inlet velocity as well as to the coarseness of the CFD grid as shown in Table 2. Nevertheless, reasonable agreement is obtained for both solids velocity and volume fraction between our finest simulation using real particles (DEM) and coarser CGPM simulations. This demonstrates the ability of this coarsegrained technique of predicting reasonably well the flow behavior in this bubbling fluidized bed. 


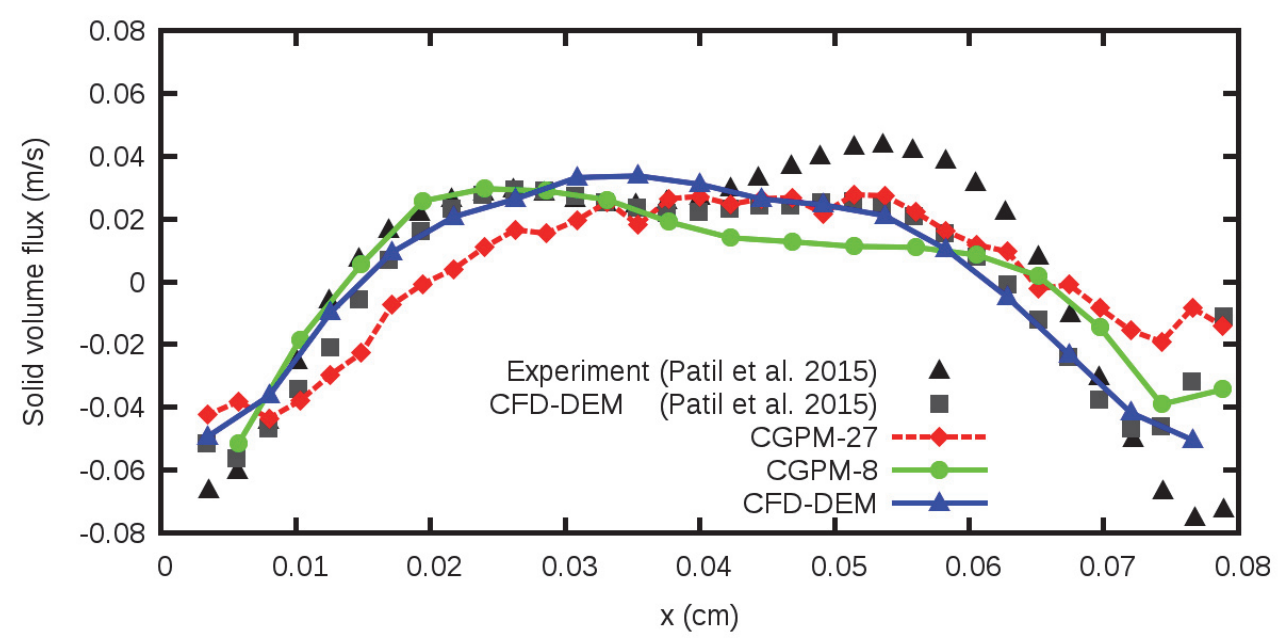

(a) Solid volume flux

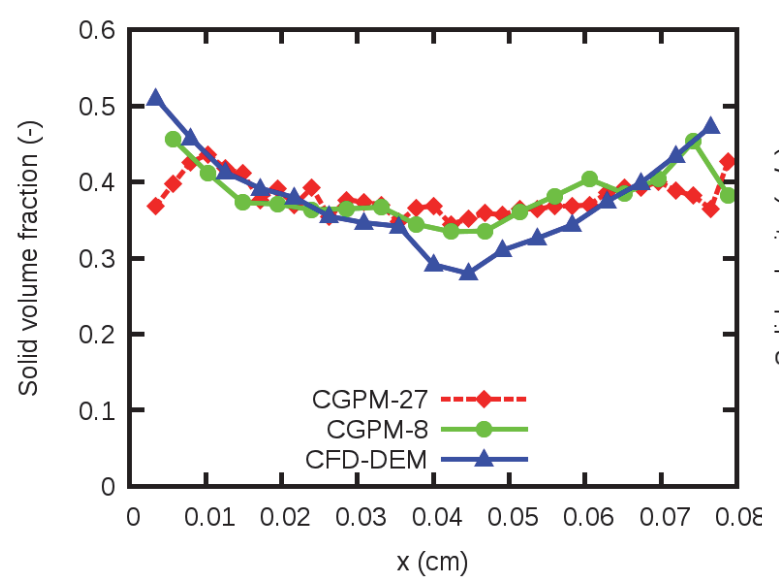

(b) Solid volume fraction

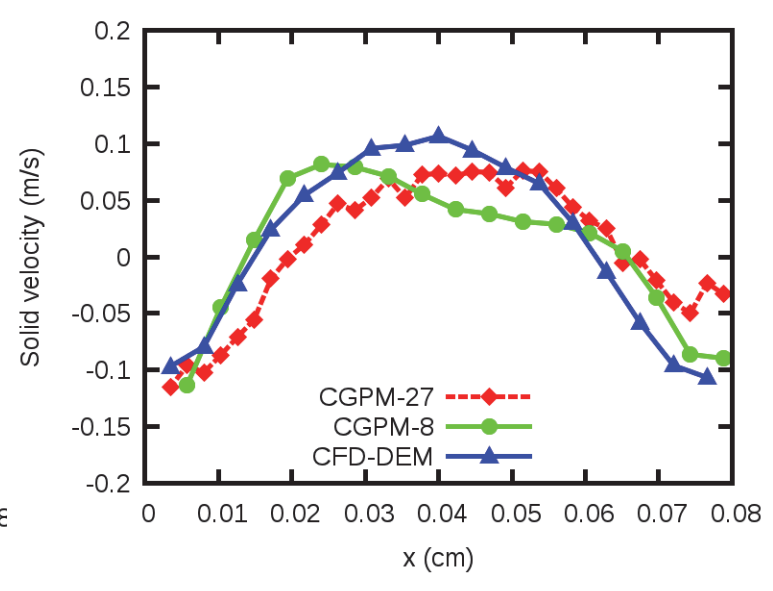

(c) Solid axial velocity

Fig. 8 Solids volumetric flux, volume fraction, and axial velocity at height $2.3 \mathrm{~cm}$ with different $W$.

\subsection{Effect of wall boundary conditions on the cooling behavior of fluidized particles}

To investigate the particle-fluid convective heat transfer, the fluidized bed system is first simulated with adiabatic wall boundary condition, which means that the heat leaves the system mainly through the outlet. If the particle-fluid convection term is calculated correctly for the CGPM, the mean particle temperature should be same as for DEM during this transient simulation.

As shown in Fig. 7, the cooling curves are almost identical for simulations with different $W$, which also compare well with previously published simulation results [35]. However, the previously published results were based on the model of Sun and Chen [26] where the total amount of heat transferred during one collision is directly calculated and added to contact particles at their first encounter instead of integrating the heat transferred during the whole DEM time step. Nevertheless, the results computed with these different models were very similar. This is because particles are well-mixed in this bubbling fluidized bed, which result in low-temperature gradient between two contacting particles. Furthermore, the contact area between two contacting particles is usually much smaller than the surface area. Intuitively, 
this means that the heat transfer through particle-particle contact can be neglected compared to the particle-fluid convection. The particle-particle heat transfer only servers as a way to homogenize temperature distribution in granular systems.

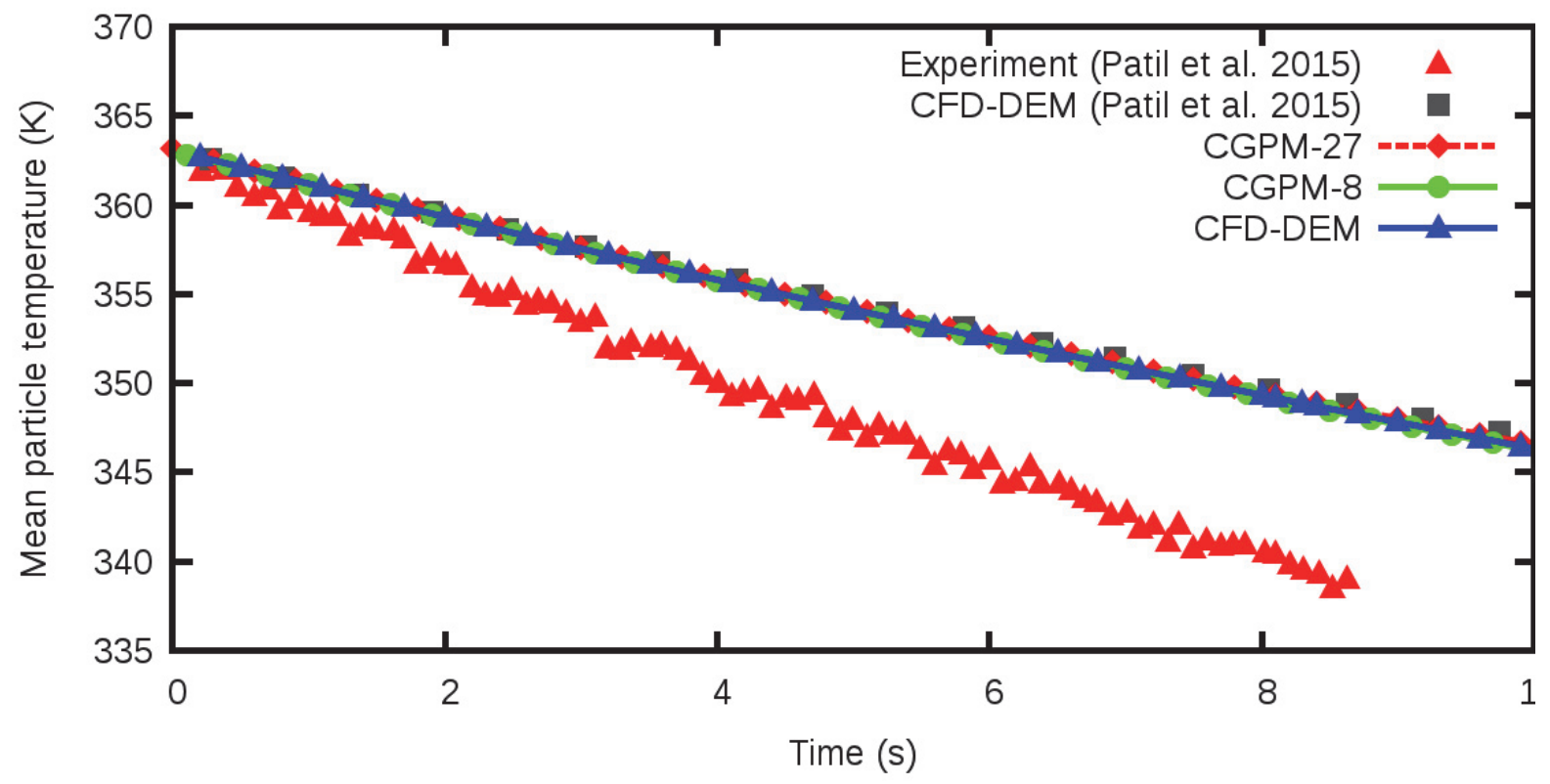

Fig. 9 Mean particle temperature profile simulated with different $W$ and adiabatic wall boundary conditions

Although our simulation results obtained with adiabatic wall boundary conditions compare well with previously published numerical results as shown in Fig. 7, the predicted cooling curves are higher than the experimental results. This is because in experiment, three plates of the fluidized bed are made of aluminum, which has a high thermal conductivity. Thus, the same fluidized bed system described earlier is simulated again with constant wall temperature boundary conditions $(293 \mathrm{~K})$. The thermal conductivity of different plates are listed in Table 1. Fig. 8 shows the mean particle temperature profile simulated with different $W$ and constant wall temperature boundary conditions of $293 \mathrm{~K}$. The cooling curves obtained by simulating a fluidized bed with different $W$ and those obtained experimentally are now in much better agreement. This means that heat losses from the aluminum plates is significant and has to be accounted for in these simulations. 


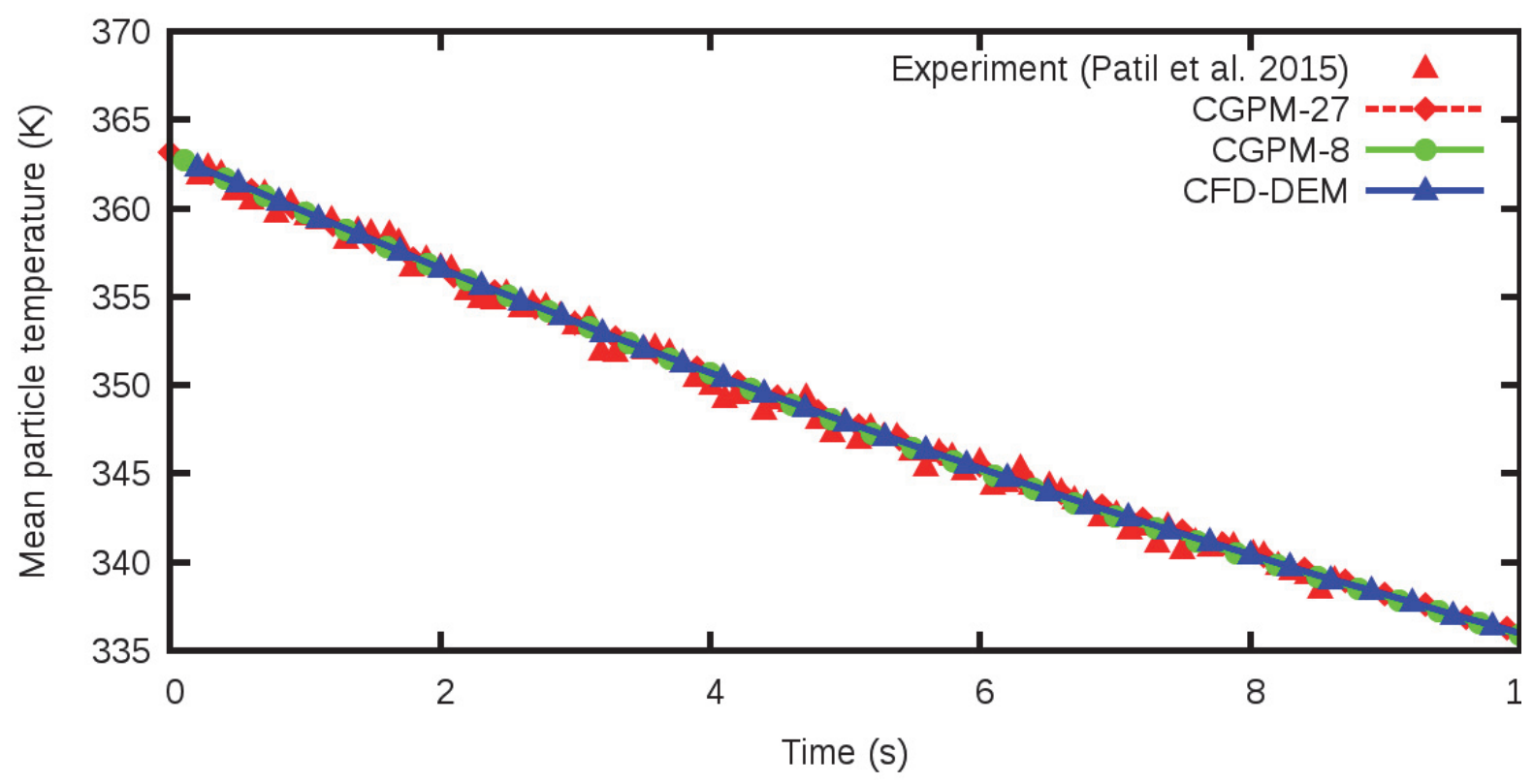

Fig. 10 Mean particle temperature profile simulated with different $W$ and constant wall temperature boundary conditions

\subsection{Quantitative analysis of different heat transfer mechanisms}

For CFD-DEM or CGPM, we can obtain particle-scale heat transfer information. Thus, it is easy to quantitatively analyze the different heat transfer mechanisms and particle temperature distribution. In fluidized beds at relatively low temperature (recall that we ignore in this study the effect of radiative heat transfer), the heat in particles is lost by particle-fluid convection, particle-fluid-wall conduction and particle-wall contact conduction. These different heat transfer mechanisms are quantitatively compared in Fig. 9 (a) using results from CFD-DEM simulation. In this figure, the heat transfer rates summed over all particles are recorded during the simulation with an average interval of $0.05 \mathrm{~s}$. The data show that the particle-fluid convection term accounts for about $65 \%$ of the total heat loss while the rest is mainly caused by particle-fluid-wall conduction. The particle-wall contact conduction term accounts for less than $1 \%$ and can, thus, be neglected in this case. These heat transfer mechanisms using different $W$ are also quantitatively compared as shown in Fig. 9 (b), (c) and (d). For the particle-fluid convection term, the results obtained using CGPM-8 are similar to CFD-DEM, while those obtained with CGPM-27 are under-predicted. This may be due to the coarser CFD grids used in case CGPM-27 as discussed in previous section. The transient profile of particle-fluid convection term decreases gradually from an initial value of about 150 watts to 100 watts after $10.0 \mathrm{~s}$. This is because as the particle temperature decreases the gradient between fluid and particle decreases as well, thus resulting in the decrease of particle-fluid convection rate. For particle-fluid-wall conduction, the results with different $W$ are very similar. This proves that our proposed model for particle-fluid-wall conduction is accurate. Also for this term, the heat transfer rate decreases from about 80 watts to 50 watts. This decrease is also due to the decrease of the temperature gradient between particle and wall. For particle-wall conduction, the heat transfer rate is less than 1 watt. Although this value is almost negligible in this case, our proposed model for particlewall conduction for CGPM works well as the results with different statistic weight are almost identical. 


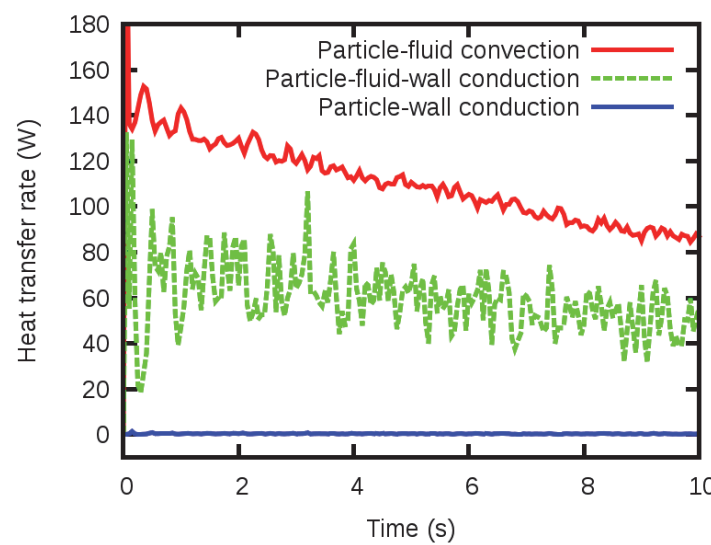

(a) Three different mechanisms

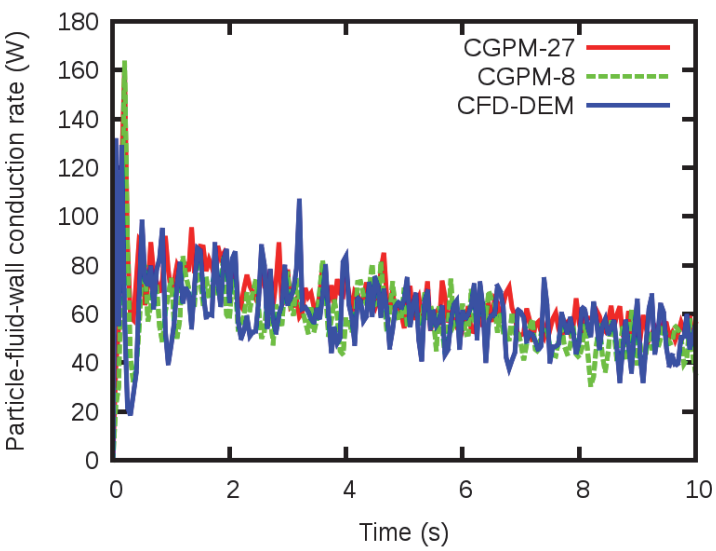

(c) particle-fluid-wall conduction

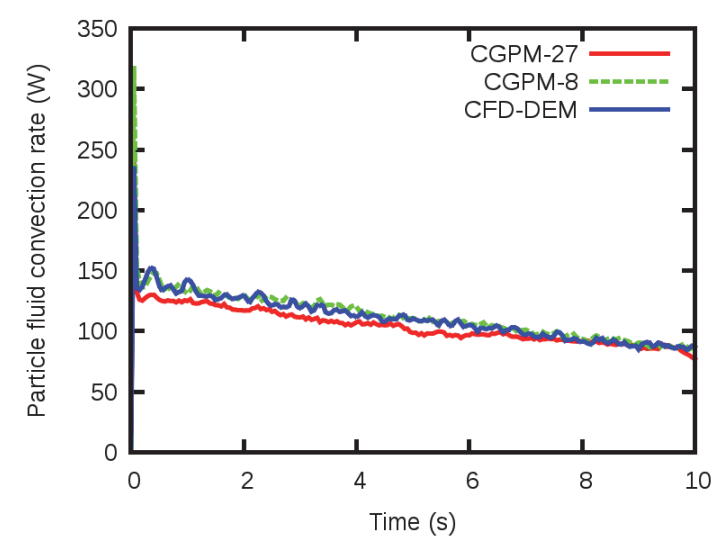

(b) Particle-fluid convection

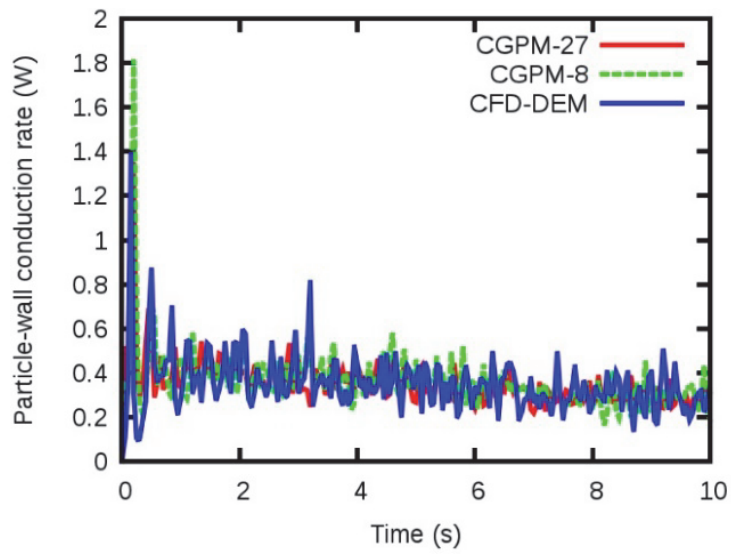

(d) particle-wall contact conduction

Fig. 11 Heat transfer mechanisms calculated with different $W$. (a) Three different mechanisms (b) particle-fluid convection; (c) particle-fluid-wall conduction; (d) particle-wall contact conduction

\subsection{Particle temperature distribution}

As mentioned before, particle scale models such as DEM and CGPM make it easy to visualize the particle temperature distribution. Fig. 10 shows two instants of particles distribution colored by their temperature at time $3.5 \mathrm{~s}$ and $5.5 \mathrm{~s}$. The particles at the bottom of the bed are quickly cooled by the inlet gas while the particles near the top of the bed have high temperature. The temperature distribution of cases CFD-DEM, CGPM-8 and CGPM- 27 are very similar with a "cool core" at the center of the bed where preferential flow of gas usually occurs. From time $3.5 \mathrm{~s}$ to $5.5 \mathrm{~s}$, all the particles are cooling down especially those at higher temperatures. For particles near the bottom gas inlet, there is little transient variation in temperature. 


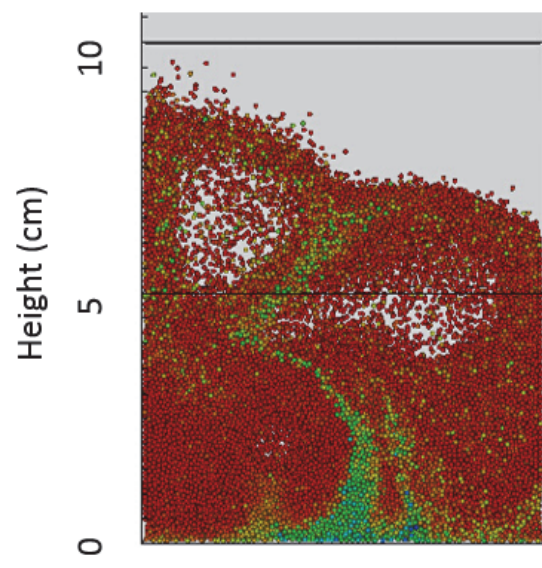

CFD-DEM

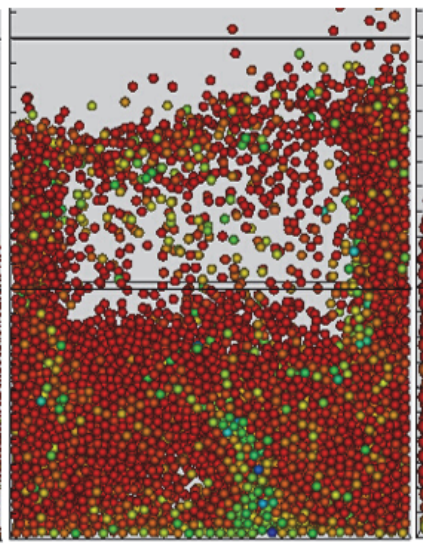

CGPM-8

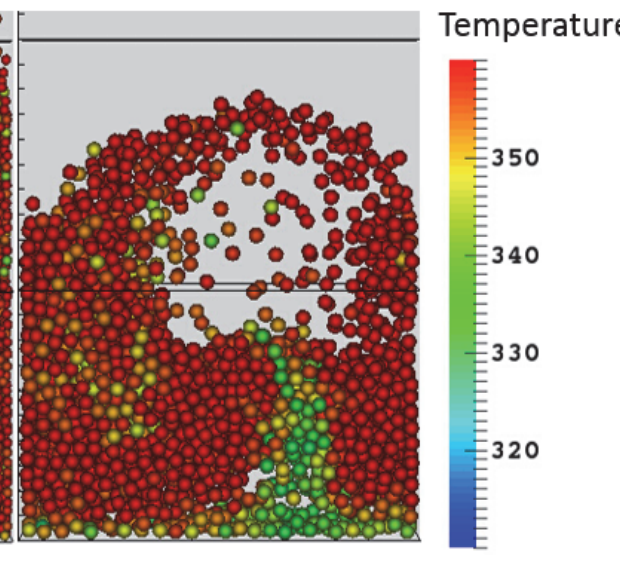

CGPM-27

(a) Time $=3.5 \mathrm{~s}$

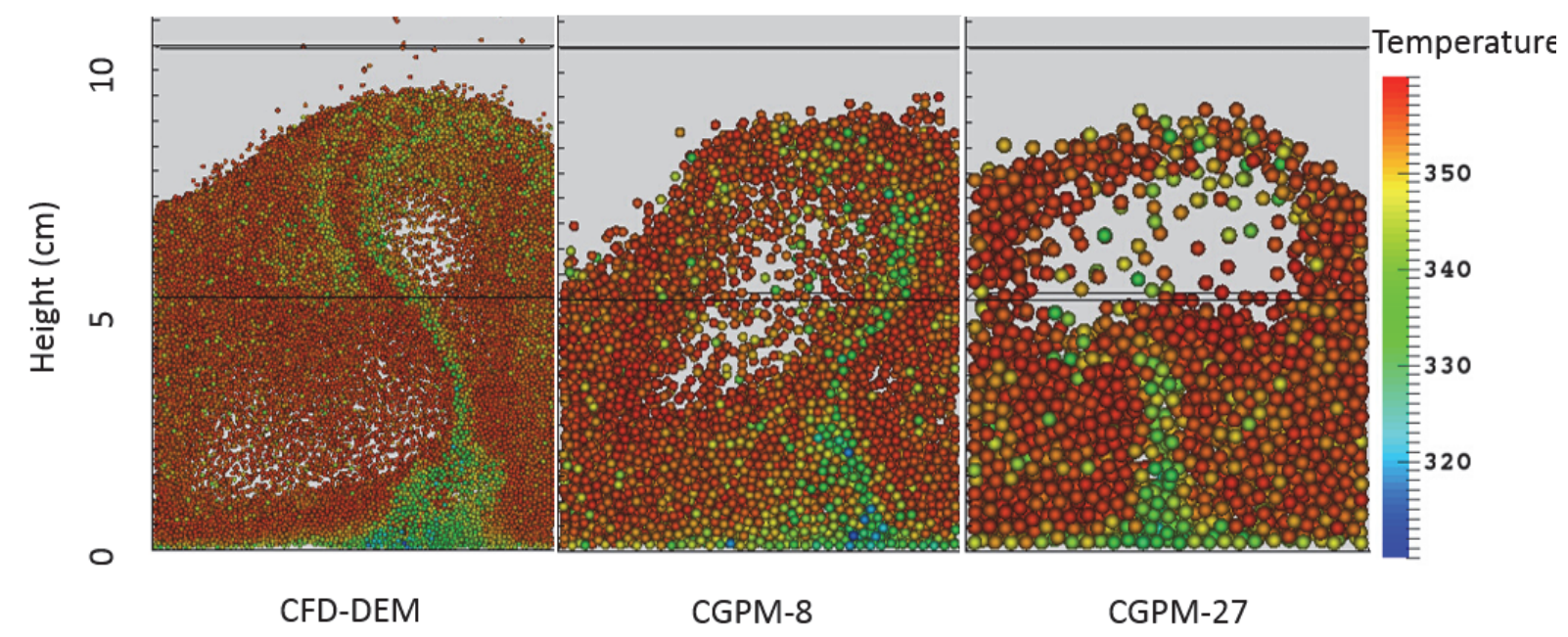

(b) Time $=5.5 \mathrm{~s}$

Fig. 12 Instantaneous particles temperature distribution with different $W$

Fig. 11 and Fig. 12 quantitatively compare the temperature distribution for all particles inside the bed at an instant calculated for different $W$ under different wall boundary conditions. Here the distribution function is defined as:

$$
F\left(T_{i}\right)=\frac{n_{i}}{N_{p} \delta_{T}}
$$

Where $n_{i}$ is number of particles with temperature in range of $T_{\mathrm{i}}-\delta_{T} / 2$ to $T_{\mathrm{i}}+\delta_{T} / 2, N_{p}$ is the total number of particles in the system. In this case, $1 \mathrm{sec}$ is used for $\delta_{\mathrm{T}}$, which is found to be small enough to resolve the distribution and large enough to contain enough particles in each segment. The narrow distribution indicates that the bubbling fluidized bed is well mixed as most of the particles have similar temperature. Also, and even for an instantaneous distribution, the proposed heat transfer model for CGPM works well as the distribution profile is quite similar between results with different $W$ and DEM. 


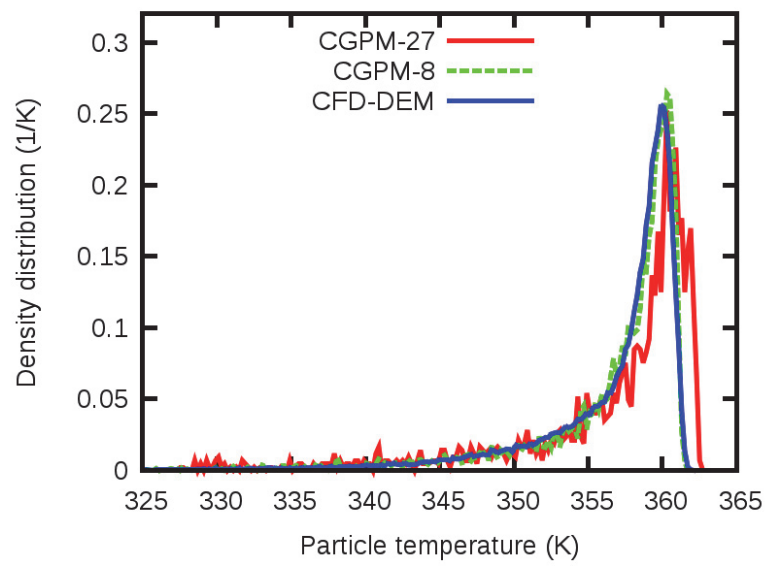

(a)

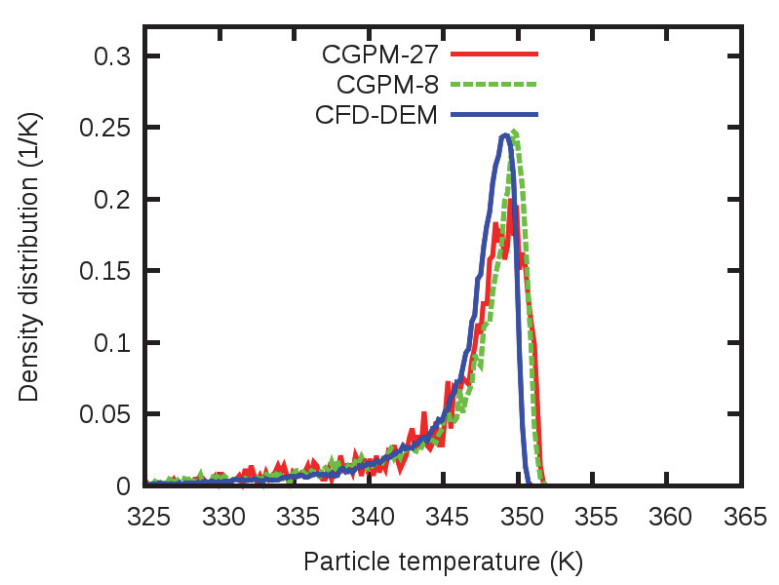

(b)

Fig. 13 Particle temperature distribution calculated with different $W$ using an adiabatic wall boundary conditions $(\mathrm{a})$ time $=3.5 \mathrm{~s}$; (b) time $=5.5 \mathrm{~s}$;

For simulations results obtained with constant wall temperature boundary conditions, the peak of the distribution profile is lower than that with adiabatic walls meaning the temperature distribution is less uniform. This is because with constant wall temperature boundary conditions the particles near this boundary will cool faster than those in the center due to the particle-fluid-wall conduction heat transfer. Also, the walls made of highly conductive material (aluminum) also increase this difference. This figure also shows that the temperature distribution profile is similar for simulation with different $W$. However, at longer time, the difference between CFD-DEM and CGPM becomes larger as shown in Fig. 12 (b). Nevertheless, such inaccuracies are not a major concern from an engineering point of view because usually the time-averaged temperature, and not an instantaneous distribution, is of interest.

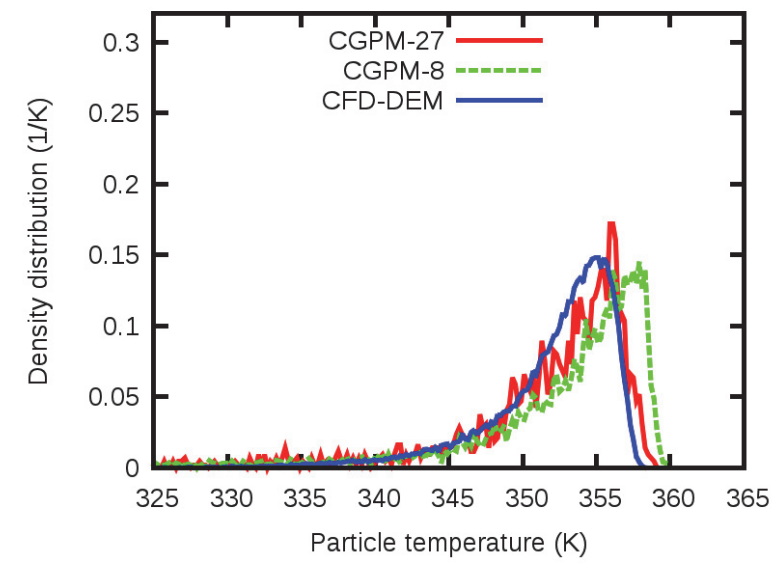

(a)

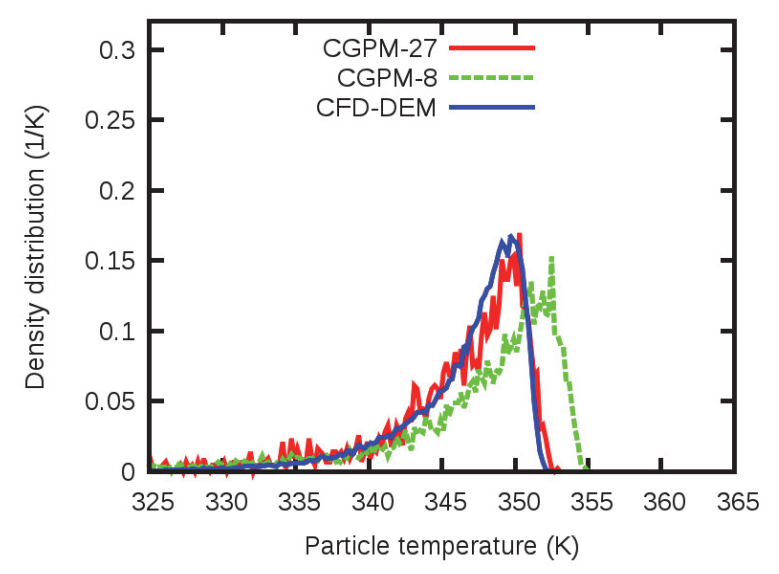

(b)

Fig. 14 Particle temperature distribution calculated with different $W$ and constant wall temperature (a) time $=3.5 \mathrm{~s}$; (b) time $=5.5 \mathrm{~s}$;

\section{Conclusions}

The CGPM has been extended to study the heat transfer in gas-solids flows. The proposed model was verified by comparing simulation results with CFD-DEM and experiment data in a gas-solids bubbling fluidized bed. The particle-fluid convection, particlefluid-wall conduction and particle-wall contact conduction terms are quantitatively analyzed. Both 
the particles volumetric flux profile and cooling curve obtained with CGPM compare well with CFD-DEM and experiment data. The particle temperature and volume fraction distribution also compare well with CFD-DEM results. The proposed model can now be used to study heat transfer in large-scale industrial gas-solids flow systems at low computation cost while maintaining an accuracy comparable to that of discrete particle models.

\section{Acknowledgements}

This research was supported in part by an appointment to the National Energy Technology Laboratory Research Participation Program, sponsored by the U.S. Department of Energy and administered by the Oak Ridge Institute for Science and Education.

\section{Disclaimer}

This report was prepared as an account of work sponsored by an agency of the United States Government. Neither the United States Government nor any agency thereof, nor any of their employees, makes any warranty, express or implied, or assumes any legal liability or responsibility for the accuracy, completeness, or usefulness of any information, apparatus, product, or process disclosed, or represents that its use would not infringe privately owned rights. Reference herein to any specific commercial product, process, or service by trade name, trademark, manufacturer, or otherwise does not necessarily constitute or imply its endorsement, recommendation, or favoring by the United States Government or any agency thereof. The views and opinions of authors expressed herein do not necessarily state or reflect those of the United States Government or any agency thereof.

\section{Appendix}

The integration results of Eq.20 and the geometry relationships:

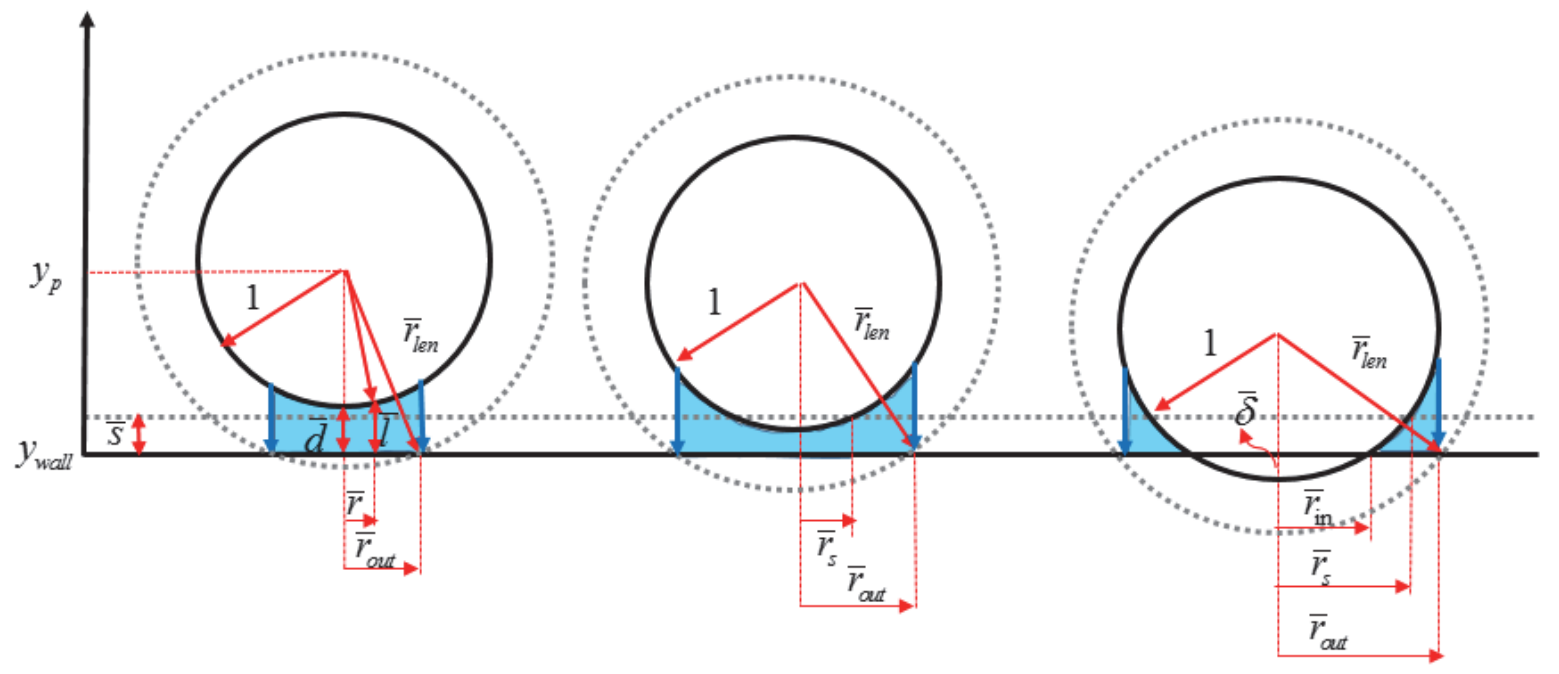
(a) $\bar{s}<\bar{d}<\bar{r}_{\text {len }}$
(b) $0<\bar{d}<\bar{s}$
(c) $\bar{d}<0$

$$
\bar{\delta}=\frac{\delta}{r_{p}}=1-\frac{y_{p}-y_{\text {wall }}}{r_{p}}
$$




$$
\begin{aligned}
& \bar{d}=-\bar{\delta} \\
& \bar{l}=1-\bar{\delta}-\sqrt{1-\bar{r}^{2}} \\
& \bar{r}_{\text {out }}=\sqrt{\bar{r}_{\text {len }}^{2}-(1-\bar{\delta})^{2}} \\
& \bar{r}_{s}=\sqrt{1-(1-\bar{\delta}-\bar{s})^{2}} \\
& \bar{r}_{i n}=\sqrt{1-(1-\bar{\delta})^{2}} \\
& \int_{\bar{r}_{\text {in }}} \frac{\bar{r}_{\text {out }}}{\bar{r}} d \bar{r}= \begin{cases}\sqrt{1-\bar{r}_{\text {out }}^{2}}+(1-\bar{\delta}) \ln \left(\frac{\left.1-\bar{\delta}-\sqrt{1-\bar{r}_{\text {out }}^{2}}\right),}{1-\bar{\delta}}\right. & \bar{s}<\bar{d}<\bar{r}_{\text {len }} \\
\frac{\bar{r}_{s}^{2}}{2 \bar{s}}+\sqrt{1-\bar{r}_{\text {out }}^{2}}-\sqrt{1-\bar{r}_{s}^{2}} & 0<\bar{d}<\bar{s} \\
+(1-\bar{\delta}) \ln \left(\frac{1-\bar{\delta}-\sqrt{1-\bar{r}_{\text {out }}^{2}}}{1-\bar{\delta}-\sqrt{1-\bar{r}_{s}^{2}},}\right. & \\
\frac{\bar{r}_{s}^{2}-\bar{r}_{\text {in }}^{2}}{2 \bar{s}}+\sqrt{1-\bar{r}_{\text {out }}^{2}}-\sqrt{1-\bar{r}_{s}^{2}} & \\
+(1-\bar{\delta}) \ln \left(\frac{1-\bar{\delta}-\sqrt{1-\bar{r}_{\text {out }}^{2}}}{1-\bar{\delta}-\sqrt{1-\bar{r}_{s}^{2}}}\right. & \bar{d}<0\end{cases}
\end{aligned}
$$

\section{Notation}

$\begin{array}{ll}d_{\mathrm{p}} & \text { diameter of particle }(\mathrm{m}) \\ d_{\mathrm{CGP}} & \text { diameter of CGP }(\mathrm{m}) \\ d_{\mathrm{x}} & \text { CFD grid size in x direction }(\mathrm{m}) \\ d_{\mathrm{y}} & \text { CFD grid size in y direction }(\mathrm{m}) \\ d_{\mathrm{z}} & \text { CFD grid size in z direction }(\mathrm{m}) \\ d_{\mathrm{CFD}} & \text { smallest CFD grid size in } \mathrm{x}, \mathrm{y} \text { and } \mathrm{z} \text { direction }(\mathrm{m}) \\ \mathrm{C}_{\mathrm{D} 0} & \text { standard drag coefficient for a CGP } \\ e_{\mathrm{CGP}} & \text { restitution coefficient of CGP } \\ \mathrm{g} & \left.\text { gravity (m/s }{ }^{2}\right) \\ \mathbf{F}_{\mathrm{c}} & \text { contact force }(\mathrm{N}) \\ \mathbf{F}_{i j}{ }^{n} & \text { normal contact force }(\mathrm{N})\end{array}$




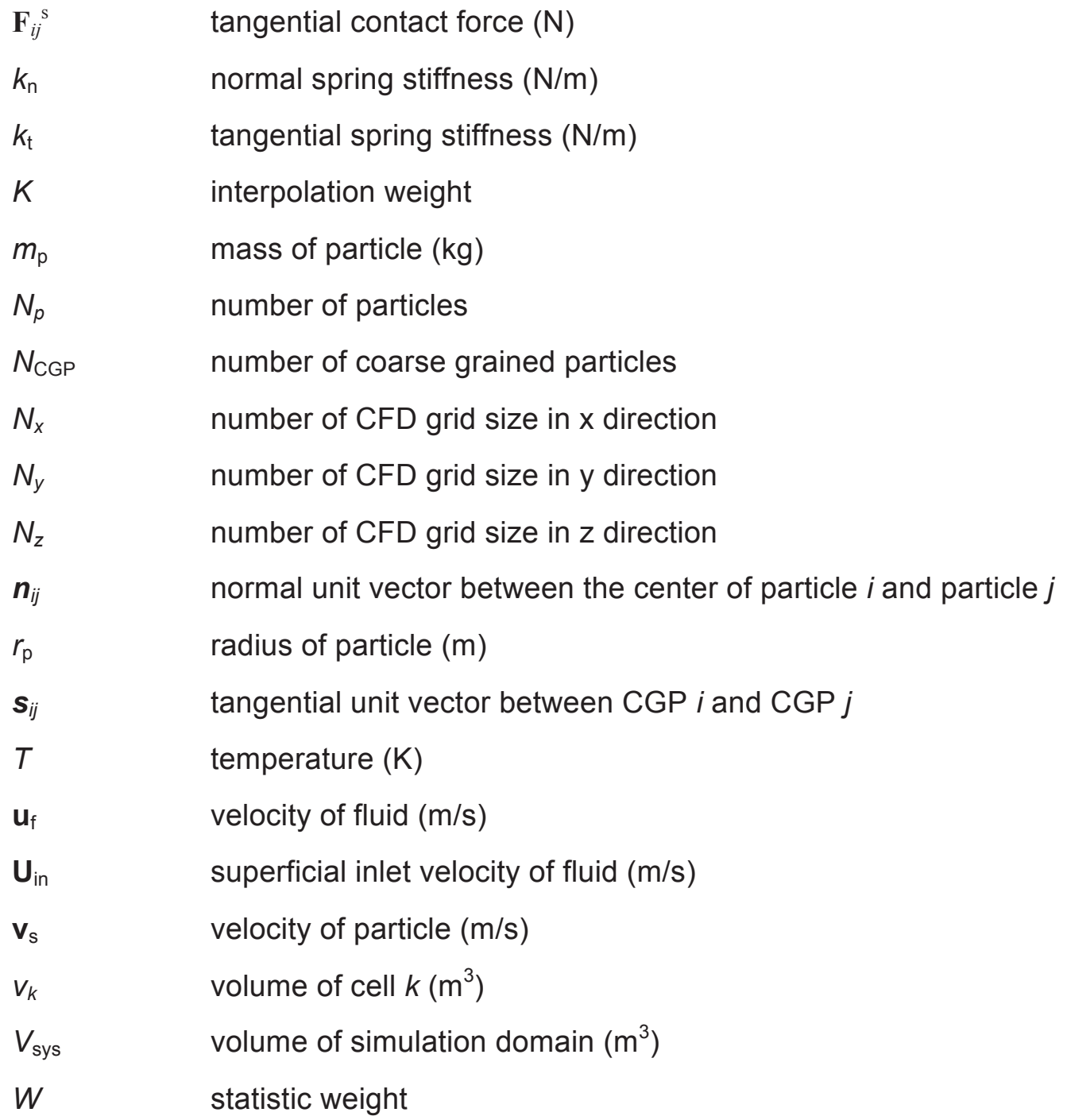

Subscripts 


$\begin{array}{ll}\mathrm{f} & \text { fluid phase } \\ \mathrm{s} & \text { solid phase } \\ \mathrm{p} & \text { particle } \\ \text { CGP } & \text { coarse-grained particle }\end{array}$

\section{References}

[1] Y. Tsuji, T. Kawaguchi, T. Tanaka, Discrete particle simulation of two-dimensional fluidized bed, Powder Technol., 77(1) (1993) 79-87.

[2] H.P. Zhu, Z.Y. Zhou, R.Y. Yang, A.B. Yu, Discrete particle simulation of particulate systems: Theoretical developments, Chem. Eng. Sci., 62(13) (2007) 3378-3396.

[3] W. Zhong, A. Yu, G. Zhou, J. Xie, H. Zhang, CFD simulation of dense particulate reaction system: Approaches, recent advances and applications, Chem. Eng. Sci., 140 (2016) 16-43.

[4] B.H. Xu, A.B. Yu, Numerical simulation of the gas-solid flow in a fluidized bed by combining discrete particle method with computational fluid dynamics, Chem. Eng. Sci., 52(16) (1997) 27852809.

[5] D.S. Nasato, C. Goniva, S. Pirker, C. Kloss, Coarse Graining for Large-scale DEM Simulations of Particle Flow - An Investigation on Contact and Cohesion Models, Procedia Engineering, 102 (2015) 1484-1490.

[6] S.C. Thakur, J.Y. Ooi, H. Ahmadian, Scaling of discrete element model parameters for cohesionless and cohesive solid, Powder Technol., 293 (2016) 130-137.

[7] M. Sakai, S. Koshizuka, Large-scale discrete element modeling in pneumatic conveying, Chem. Eng. Sci., 64(3) (2009) 533-539.

[8] J.E. Hilton, P.W. Cleary, Comparison of non-cohesive resolved and coarse grain DEM models for gas flow through particle beds, Appl. Math. Model., 38(17-18) (2014) 4197-4214.

[9] M. Sakai, M. Abe, Y. Shigeto, S. Mizutani, H. Takahashi, A. Viré, J.R. Percival, J. Xiang, C.C. Pain, Verification and validation of a coarse grain model of the DEM in a bubbling fluidized bed, Chem. Eng. J., 244 (2014) 33-43.

[10] K. Chu, J. Chen, A. Yu, Applicability of a coarse-grained CFD-DEM model on dense medium cyclone, Miner. Eng., 90 (2016) 43-54.

[11] L. Lu, J. Xu, W. Ge, Y. Yue, X. Liu, J. Li, EMMS-based discrete particle method (EMMS-DPM) for simulation of gas-solid flows, Chem. Eng. Sci., 120(0) (2014) 67-87.

[12] L. Lu, J. Xu, W. Ge, G. Gao, Y. Jiang, M. Zhao, X. Liu, J. Li, Computer virtual experiment on fluidized beds using a coarse-grained discrete particle method-EMMS-DPM, Chem. Eng. Sci., 155 (2016) 314-337.

[13] L. Lu, K. Yoo, S. Benyahia, Coarse-Grained-Particle Method for Simulation of Liquid-Solids Reacting Flows, Ind. Eng. Chem. Res., (2016).

[14] M. Syamlal, W. Rogers, T.J. O'Brien, MFIX documentation: Theory guide, National Energy Technology Laboratory, Department of Energy, 1993.

[15] S. Benyahia, On the Effect of Subgrid Drag Closures, Ind. Eng. Chem. Res., 49(11) (2010) $5122-$ 5131.

[16] N. Yang, W. Wang, W. Ge, J. Li, CFD simulation of concurrent-up gas-solid flow in circulating fluidized beds with structure-dependent drag coefficient, Chem. Eng. J., 96(1-3) (2003) 71-80.

[17] W. Wang, J. Li, Simulation of gas-solid two-phase flow by a multi-scale CFD approach-of the EMMS model to the sub-grid level, Chem. Eng. Sci., 62(1-2) (2007) 208-231.

[18] Y. Igci, A.T. Andrews, S. Sundaresan, S. Pannala, T. O'Brien, Filtered two-fluid models for fluidized gas-particle suspensions, AIChE J., 54(6) (2008) 1431-1448.

[19] R. Beetstra, M.A. van der Hoef, J.A.M. Kuipers, Drag force of intermediate Reynolds number flow past mono- and bidisperse arrays of spheres, AlChE J., 53(2) (2007) 489-501.

[20] R. Garg, J. Galvin, T. Li, S. Pannala, Documentation of open-source MFIX-DEM software for gassolids flows, in: https://mfix.netl.doe.gov/download/mfix/mfix_current_documentation/dem_doc_2012-1.pdf, 2012.

[21] R. Garg, J. Galvin, T. Li, S. Pannala, Open-source MFIX-DEM software for gas-solids flows: Part I-Verification studies, Powder Technol., 220 (2012) 122-137.

[22] T. Li, R. Garg, J. Galvin, S. Pannala, Open-source MFIX-DEM software for gas-solids flows: Part II - Validation studies, Powder Technol., 220 (2012) 138-150. 
[23] S. Benyahia, J.E. Galvin, Estimation of Numerical Errors Related to Some Basic Assumptions in Discrete Particle Methods, Ind. Eng. Chem. Res., 49(21) (2010) 10588-10605.

[24] D. Gidaspow, Multiphase flow and fluidization, continuum and kinetic theory descriptions, Academic Press, San Diego, (1994).

[25] C.K.K. Lun, S.B. Savage, D.J. Jeffrey, N. Chepurniy, Kinetic theories for granular flow: inelastic particles in Couette flow and slightly inelastic particles in a general flow field, J. Fluid Mech., 140 (1984) 223-256.

[26] J. Sun, M.M. Chen, A theoretical analysis of heat transfer due to particle impact, Int. J. Heat Mass Transfer, 31(5) (1988) 969-975.

[27] G.K. Batchelor, R.W. Brien, Thermal or Electrical Conduction Through a Granular Material, Proceedings of the Royal Society of London. A. Mathematical and Physical Sciences, 355(1682) (1977) 313.

[28] J. Musser, Modeling of Heat Transfer and Reactive Chemistry for Particles in Gas-solid Flow Utilizing Continuum-discrete Methodology (CDM), Ph.D., West Virginia University, Morgantown, WV, US, 2011.

[29] G.J. Cheng, A.B. Yu, P. Zulli, Evaluation of effective thermal conductivity from the structure of a packed bed, Chem. Eng. Sci., 54(19) (1999) 4199-4209.

[30] Z.Y. Zhou, A.B. Yu, P. Zulli, Particle scale study of heat transfer in packed and bubbling fluidized beds, AlChE J., 55(4) (2009) 868-884.

[31] D. Rong, M. Horio, DEM simulation of char combustion in a fluidized bed, in: Second International Conference on CFD in the Minerals and Process Industries CSIRO, Melbourne, Australia, 1999, pp. 65-70.

[32] D.J. Gunn, Transfer of heat or mass to particles in fixed and fluidised beds, Int. J. Heat Mass Transfer, 21(4) (1978) 467-476.

[33] K.A. Buist, L.J.H. Seelen, N.G. Deen, J.T. Padding, J.A.M. Kuipers, On an efficient hybrid soft and hard sphere collision integration scheme for DEM, Chem. Eng. Sci., 153 (2016) 363-373.

[34] Z.Y. Zhou, A.B. Yu, P. Zulli, A new computational method for studying heat transfer in fluid bed reactors, Powder Technol., 197(1-2) (2010) 102-110.

[35] A.V. Patil, E.A.J.F. Peters, J.A.M. Kuipers, Comparison of CFD-DEM heat transfer simulations with infrared/visual measurements, Chem. Eng. J., 277 (2015) 388-401. 